MATHEMATICS OF COMPUTATION

Volume 75 , Number 254, Pages 791-815

S 0025-5718(05)01801-6

Article electronically published on November 30, 2005

\title{
STRUCTURED PRECONDITIONERS FOR NONSINGULAR MATRICES OF BLOCK TWO-BY-TWO STRUCTURES
}

\author{
ZHONG-ZHI BAI
}

\begin{abstract}
For the large sparse block two-by-two real nonsingular matrices, we establish a general framework of practical and efficient structured preconditioners through matrix transformation and matrix approximations. For the specific versions such as modified block Jacobi-type, modified block GaussSeidel-type, and modified block unsymmetric (symmetric) Gauss-Seidel-type preconditioners, we precisely describe their concrete expressions and deliberately analyze eigenvalue distributions and positive definiteness of the preconditioned matrices. Also, we show that when these structured preconditioners are employed to precondition the Krylov subspace methods such as GMRES and restarted GMRES, fast and effective iteration solvers can be obtained for the large sparse systems of linear equations with block two-by-two coefficient matrices. In particular, these structured preconditioners can lead to efficient and high-quality preconditioning matrices for some typical matrices from the real-world applications.
\end{abstract}

\section{INTRODUCTION}

Let $\mathbb{R}^{n}$ represent the real $n$-dimensional vector space, and $\mathbb{R}^{n \times n}$ the real $n \times n$ matrix space. Consider an iterative solution of the large sparse system of linear equations

$$
A x=b, \quad A \in \mathbb{R}^{n \times n} \text { nonsingular and } x, b \in \mathbb{R}^{n} .
$$

In this paper, we will study algorithmic constructions and theoretical properties of practical and efficient structured preconditioners to the matrix $A \in \mathbb{R}^{n \times n}$ which is of the block two-by-two structure

$$
A=\left[\begin{array}{ll}
B & E \\
F & C
\end{array}\right],
$$

where $B \in \mathbb{R}^{p \times p}$ nonsingular, $C \in \mathbb{R}^{q \times q}, E \in \mathbb{R}^{p \times q}$ and $F \in \mathbb{R}^{q \times p}$, with $p \geq q$, such that $A \in \mathbb{R}^{n \times n}$ is nonsingular. Evidently, when the matrix block $B$ is nonsingular, the matrix $A$ is nonsingular if and only if its Schur complement $S_{A}=C-F B^{-1} E$ is nonsingular.

Received by the editor October 16, 2003 and, in revised form, March 16, 2005.

2000 Mathematics Subject Classification. Primary 65F10, 65F50.

Key words and phrases. Block two-by-two matrix, preconditioner, modified block relaxation iteration, eigenvalue distribution, positive definiteness.

Subsidized by The Special Funds For Major State Basic Research Projects (No. G1999032803), The National Basic Research Program (No. 2005CB321702), and The National Natural Science Foundation (No. 10471146), P.R. China. 
Linear systems of the form (1.1)-(1.2) arise in a variety of scientific and engineering applications, including computational fluid dynamics [21, 23, 26], mixed finite element approximation of elliptic partial differential equations [16, 38, optimization 25, 30, 34, optimal control [13, weighted and equality constrained least squares estimation [14, stationary semiconductor device [36, 42, 43], structural analysis [44, electrical networks [44], inversion of geophysical data [31], and so on.

As we have known, preconditioned Krylov subspace methods 40 are efficient iterative solvers for the system of linear equations (1.1)-(1.2), and effective and high-quality preconditioners play a crucial role to guarantee their fast convergence and economical costs. A number of structured preconditioners have been studied in the literature for some special cases of the block two-by-two matrix $A$ in (1.2). Besides specialized incomplete factorization preconditioners [17, 18] we mention, among others, algebraic multilevel iteration preconditioners [2, 3, 4, 5, 12, block and approximate Schur complement preconditioners [21, 23], splitting iteration preconditioners [15, 19, 22, 28, 29, 39, 45, block definite and indefinite preconditioners [24, 34, 38, 10], and block triangular preconditioners [35, 37, 10]. Theoretical analyses and experimental results have shown that these preconditioners may lead to nicely clustered eigenvalue distributions of the preconditioned matrices and, hence, result in fast convergence of the preconditioned Krylov subspace iteration methods for solving the large sparse system of linear equations (1.1)-(1.2). However, exact inversions of the matrix block $B$ or $C$, as well as the Schur complement $S_{A}$, are demanded for most of these preconditioners, which makes them less practical and effective in actual applications.

In this paper, by sufficiently utilizing the matrix structure and property, we first establish a general framework of a class of practical and efficient structured preconditioners to the matrix $A \in \mathbb{R}^{n \times n}$ in (1.2) through matrix transformation and several steps of matrix approximations; these preconditioners can avoid the exact inversions of the matrix blocks $B$ and $C$, as well as the Schur complement $S_{A}$, and cover the known preconditioners mentioned previously as special cases. Then, with this framework we further present a family of practical and efficient preconditioners by technically combining it with the modified block relaxation iterations 6. 7, which includes the modified block Jacobi-type, the modified block GaussSeidel-type and the modified block unsymmetric (symmetric) Gauss-Seidel-type preconditioners as typical examples. Moreover, we particularly discuss the eigenvalue distributions and the positive definiteness of the preconditioned matrices with respect to the modified block Jacobi-type, the modified block Gauss-Seidel-type, and the modified block unsymmetric (symmetric) Gauss-Seidel-type preconditioners to the block two-by-two matrix $A$, and deliberately address the applications of these preconditioners to three classes of real-world matrices, i.e., the symmetric positive definite matrix, the saddle point matrix and the Hamiltonian matrix. Besides, we show that when these structured preconditioners are employed to precondition the Krylov subspace methods such as GMRES or restarted GMRES, fast and effective iteration solvers can be obtained for the large sparse system of linear equations (1.1)-(1.2).

The organization of this paper is as follows. After establishing the general framework of the structured preconditioners in Section 2, we present the modified block splitting iteration preconditioners and study the eigenvalue distributions and the positive definiteness of the corresponding preconditioned matrices in Section 3. 
connections of these preconditioners to Krylov subspace iteration methods are also briefly discussed in this section. Specifications of these preconditioners to three classes of real-world matrices are investigated in Section [4. Finally, in Section 5 , we use a brief conclusion and several remarks to end the paper.

\section{General framework of the structured preconditioners}

The construction of our structured preconditioners basically includes the following three steps: Firstly, seek two nonsingular block two-by-two matrices $P, Q \in$ $\mathbb{R}^{n \times n}$ such that $P$ and $Q$ are easily invertible and $A=P H Q$ holds for a block two-by-two matrix $H \in \mathbb{R}^{n \times n}$ of certain good properties; secondly, approximate the matrix $H$ by another block two-by-two matrix $\bar{W} \in \mathbb{R}^{n \times n}$ by dropping some higher-order small block quantities; thirdly, approximate the matrix $\bar{W}$ further by another block two-by-two matrix $W \in \mathbb{R}^{n \times n}$ that is also easily invertible. Then, the resulting preconditioners are of the form $M=P W Q$. See [9, 11].

Let $L_{B}, R_{B} \in \mathbb{R}^{p \times p}$ and $L_{C}, R_{C} \in \mathbb{R}^{q \times q}$ be nonsingular matrices such that

$$
L_{B}^{-1} B R_{B}^{-1}=J_{B} \quad \text { and } \quad L_{C}^{-1} C R_{C}^{-1}=J_{C},
$$

or equivalently,

$$
B=L_{B} J_{B} R_{B} \quad \text { and } \quad C=L_{C} J_{C} R_{C},
$$

where $J_{B} \in \mathbb{R}^{p \times p}$ is a matrix approximating the identity matrix $I_{B} \in \mathbb{R}^{p \times p}$, and $J_{C} \in \mathbb{R}^{q \times q}$ is a matrix approximating the identity matrix $I_{C} \in \mathbb{R}^{q \times q}$ when it is positive definite and approximating $-I_{C} \in \mathbb{R}^{q \times q}$ when it is negative definite. For simplicity, in the sequel we will abbreviate the identity matrices $I_{B}$ and $I_{C}$ as $I$, with their dimensions being inferred from the context.

Evidently, $L_{B}, R_{B}$ and $L_{C}, R_{C}$ can be considered as split preconditioners to the matrix blocks $B$ and $C$, respectively, whose preconditioning properties can be measured by the approximation degrees of the matrices $J_{B}$ and $\pm J_{C}$ to the identity matrix $I$. There are many possible choices of the matrices $L_{B}, R_{B}$ and $L_{C}, R_{C}$. For example, they may be the incomplete lower-upper triangular factors 2. 40, the incomplete orthogonal triangular factors [8, the approximate inverse preconditioners [40, the splitting iteration matrices [2, 6, 7, 27, the multigrid or the algebraic multilevel approximations [2, 3, 4, 5, 12, or even technical combinations of the above-mentioned matrices, to the matrix blocks $B$ and $C$, respectively.

In particular, when $C \in \mathbb{R}^{q \times q}$ is singular, besides the possible choices mentioned above, we may choose $L_{C}$ and $R_{C}$ according to the following cases:

(i) If $C$ is a symmetric positive semidefinite matrix, we may let $L_{C}=R_{C}=I$. Hence, $J_{C}=C$ is also symmetric positive semidefinite.

(ii) If $C$ is a symmetric negative semidefinite matrix, we may let $L_{C}=-I$ and $R_{C}=I$ (or $L_{C}=I$ and $R_{C}=-I$ ). Hence, $J_{C}=-C$ is symmetric positive semidefinite. Or we may let $L_{C}=R_{C}=I$. Hence, $J_{C}=C$ is also symmetric negative semidefinite.

(iii) If $C$ is a general singular matrix, we may let $L_{C}=R_{C}=I$. Hence, $J_{C}=C$ is also singular.

To construct a high-quality structured preconditioner to the block two-by-two matrix $A \in \mathbb{R}^{n \times n}$, we introduce matrices

$$
D_{L}=\left[\begin{array}{cc}
L_{B} & O \\
O & L_{C}
\end{array}\right], \quad D_{R}=\left[\begin{array}{cc}
R_{B} & O \\
O & R_{C}
\end{array}\right]
$$


and

$$
\bar{E}=L_{B}^{-1} E R_{C}^{-1}, \quad \bar{F}=L_{C}^{-1} F R_{B}^{-1},
$$

where $O$ denotes the zero matrix. Then from (2.2) we have

$$
\begin{aligned}
A & =\left[\begin{array}{cc}
B & E \\
F & C
\end{array}\right]=\left[\begin{array}{cc}
L_{B} J_{B} R_{B} & E \\
F & L_{C} J_{C} R_{C}
\end{array}\right] \\
& =\left[\begin{array}{cc}
L_{B} & O \\
O & L_{C}
\end{array}\right]\left[\begin{array}{cc}
J_{B} & L_{B}^{-1} E R_{C}^{-1} \\
L_{C}^{-1} F R_{B}^{-1} & J_{C}
\end{array}\right]\left[\begin{array}{cc}
R_{B} & O \\
O & R_{C}
\end{array}\right] \\
& :=D_{L} \bar{A} D_{R},
\end{aligned}
$$

where

$$
\bar{A}:=\left[\begin{array}{cc}
J_{B} & \bar{E} \\
\bar{F} & J_{C}
\end{array}\right]
$$

Furthermore, we can find a unit lower triangular matrix $L \in \mathbb{R}^{n \times n}$ and a unit upper triangular matrix $U \in \mathbb{R}^{n \times n}$ of block two-by-two structures such that $H=$ $L \bar{A} U$ is block-diagonally dominant as far as possible and may also possess some other desired good properties.

In fact, if we let

$$
L=\left[\begin{array}{cc}
I & O \\
L_{21} & I
\end{array}\right] \text { and } U=\left[\begin{array}{cc}
I & U_{12} \\
O & I
\end{array}\right]
$$

then by concrete computations we obtain

$$
\begin{aligned}
H & =\left[\begin{array}{cc}
H_{11} & H_{12} \\
H_{21} & H_{22}
\end{array}\right]:=L \bar{A} U \\
& =\left[\begin{array}{cc}
I & O \\
L_{21} & I
\end{array}\right]\left[\begin{array}{cc}
J_{B} & \bar{E} \\
\bar{F} & J_{C}
\end{array}\right]\left[\begin{array}{cc}
I & U_{12} \\
O & I
\end{array}\right]
\end{aligned}
$$

with

$$
\begin{cases}H_{11}=J_{B}, & H_{12}=J_{B} U_{12}+\bar{E} \\ H_{21}=L_{21} J_{B}+\bar{F}, & H_{22}=J_{C}+L_{21} J_{B} U_{12}+L_{21} \bar{E}+\bar{F} U_{12},\end{cases}
$$

and

$$
A=D_{L} \bar{A} D_{R}=D_{L}\left(L^{-1} H U^{-1}\right) D_{R}=\left(D_{L} L^{-1}\right) H\left(U^{-1} D_{R}\right):=P H Q
$$

with

$$
P:=D_{L} L^{-1}=\left[\begin{array}{cc}
L_{B} & O \\
O & L_{C}
\end{array}\right]\left[\begin{array}{cc}
I & O \\
-L_{21} & I
\end{array}\right]=\left[\begin{array}{cc}
L_{B} & O \\
-L_{C} L_{21} & L_{C}
\end{array}\right]
$$

and

$$
Q:=U^{-1} D_{R}=\left[\begin{array}{cc}
I & -U_{12} \\
O & I
\end{array}\right]\left[\begin{array}{cc}
R_{B} & O \\
O & R_{C}
\end{array}\right]=\left[\begin{array}{cc}
R_{B} & -U_{12} R_{C} \\
O & R_{C}
\end{array}\right] .
$$

We can now choose the matrices $L$ and $U$ such that either of the following two principles is satisfied as far as possible:

$\left(P_{1}\right)$ the matrix $H$ is block-diagonally dominant and symmetric;

$\left(P_{2}\right)$ the matrix $H$ is block-diagonally dominant and skew-symmetric. 
This is because if the matrix $H$ satisfies either of the principles $\left(P_{1}\right)$ and $\left(P_{2}\right)$, we can easily construct a good approximation to it, and hence, obtain a high-quality preconditioner $M$ to the original matrix $A$.

According to both $\left(P_{1}\right)$ and $\left(P_{2}\right)$, we can take $L_{21}$ and $U_{12}$ such that

$$
\left\{\begin{array}{l}
H_{21}=L_{21} J_{B}+\bar{F} \approx\left(J_{B} U_{12}+\bar{E}\right)^{T}= \pm H_{12}^{T} \\
H_{21}=L_{21} J_{B}+\bar{F} \approx O
\end{array}\right.
$$

Recalling that $J_{B} \approx I$, we can let

$$
L_{21}=-\bar{F} \quad \text { and } \quad U_{12}=-\bar{E} .
$$

Thus, for both cases, it follows from (2.4) and (2.5) that the matrices $P$ and $Q$ have the following expressions:

$$
P=\left[\begin{array}{cc}
L_{B} & O \\
L_{C} \bar{F} & L_{C}
\end{array}\right], \quad Q=\left[\begin{array}{cc}
R_{B} & \bar{E} R_{C} \\
O & R_{C}
\end{array}\right]
$$

Therefore, for these choices of the matrices $P$ and $Q$, we have

$$
\begin{aligned}
H & =\left[\begin{array}{cc}
J_{B} & \left(I-J_{B}\right) \bar{E} \\
\bar{F}\left(I-J_{B}\right) & J_{C}-\overline{F E}-\bar{F}\left(I-J_{B}\right) \bar{E}
\end{array}\right] \\
& \approx\left[\begin{array}{cc}
J_{B} & \left(I-J_{B}\right) \bar{E} \\
\bar{F}\left(I-J_{B}\right) & J_{C}-\overline{F E}
\end{array}\right]:=\bar{W} .
\end{aligned}
$$

Because the nonsingularity of the matrix $A$ implies that the matrix $\bar{A}$ and its Schur complement $S_{\bar{A}}:=J_{C}-\bar{F} J_{B}^{-1} \bar{E}$ are nonsingular, and

$$
J_{C}-\overline{F E}=S_{\bar{A}}+\bar{F}\left(I-J_{B}\right) J_{B}^{-1} \bar{E}
$$

and the Schur complement of $\bar{W}$ is

$$
S_{\bar{W}}:=J_{C}-\overline{F E}-\bar{F}\left(I-J_{B}\right) J_{B}^{-1}\left(I-J_{B}\right) \bar{E}=S_{\bar{A}}-\bar{F}\left(I-J_{B}\right) J_{B}^{-1} \bar{E},
$$

we immediately know that when

$$
\left\|I-J_{B}\right\|_{2}<\max \left\{\frac{1}{1+\|\bar{E}\|_{2}\left\|S_{\bar{A}}^{-1} \bar{F}\right\|_{2}}, \quad \frac{1}{1+\left\|\bar{E} S_{\bar{A}}^{-1}\right\|_{2}\|\bar{F}\|_{2}}\right\},
$$

both matrices $J_{C}-\overline{F E}$ and $\bar{W}$ are nonsingular.

Now, if we let $W \in \mathbb{R}^{n \times n}$ be a nonsingular "replacement" of the matrix $\bar{W}$, or in other words, a "replacement" to the matrix $H$, then the matrix

$$
M=P W Q
$$

is a natural preconditioner to the original matrix $A \in \mathbb{R}^{n \times n}$, and under the condition (2.9) this preconditioner is well defined.

Note that here we use the term "replacement" rather than "approximation". This is because sometimes we may choose the matrix $W$ being not an approximation to $\bar{W}$ in the usual sense so that the obtained preconditioner and the preconditioned matrix can possess some desired properties such as positive definiteness and, hence, a specified Krylov subspace iteration method may exploit its efficiency sufficiently.

If $M$ is used as a left preconditioner to $A$, then

$$
M^{-1} A=(P W Q)^{-1}(P H Q)=Q^{-1}\left(W^{-1} H\right) Q:=Q^{-1} K_{L} Q
$$

with

$$
K_{L}=W^{-1} H
$$


Therefore, the preconditioning property of $M$ to $A$ is determined by the properties of the matrices $K_{L}$ and $Q$. If $M$ is used as a right preconditioner to $A$, then

$$
A M^{-1}=(P H Q)(P W Q)^{-1}=P\left(H W^{-1}\right) P^{-1}:=P K_{R} P^{-1}
$$

with

$$
K_{R}=H W^{-1} .
$$

Therefore, the preconditioning property of $M$ to $A$ is determined by the properties of the matrices $K_{R}$ and $P$. In general, if the matrix $W$ admits a split form

$$
W=W_{L} W_{R},
$$

then (2.10) straightforwardly leads to a split preconditioner

$$
M=\left(P W_{L}\right)\left(W_{R} Q\right):=M_{L} M_{R}, \quad \text { with } \quad M_{L}=P W_{L} \quad \text { and } \quad M_{R}=W_{R} Q
$$

to the original matrix $A$. Because

$$
M_{L}^{-1} A M_{R}^{-1}=\left(P W_{L}\right)^{-1}(P H Q)\left(W_{R} Q\right)^{-1}=W_{L}^{-1} H W_{R}^{-1}:=K,
$$

we see that the preconditioning property of $M$ to $A$ is determined by the property of the matrix $K$.

Evidently, the matrices $K_{L}, K_{R}$ and $K$ are similar, and hence, they have exactly the same spectrum. However, the eigenvectors of these kinds of preconditioned matrices are usually quite different, which may lead to different performance results of the corresponding preconditioned Krylov subspace iteration methods.

In actual applications, when the matrix $M$ defined in (2.10) is employed as a preconditioner to some Krylov subspace iteration method for solving the block two-by-two system of linear equations (1.1), we need to solve a generalized residual equation of the form

$$
M z=r
$$

at each iteration step, where $r$ is the current residual vector. By making use of the two-by-two block structure of $M$, we can obtain the following practical procedure for computing the generalized residual vector $z=M^{-1} r$.

Procedure for computing the generalized residual vector. Let $r=\left(r_{1}^{T}, r_{2}^{T}\right)^{T}$, $z=\left(z_{1}^{T}, z_{2}^{T}\right)^{T}$ and $w=\left(w_{1}^{T}, w_{2}^{T}\right)^{T}$, with $r_{1}, z_{1}, w_{1} \in \mathbb{R}^{p}$ and $r_{2}, z_{2}, w_{2} \in \mathbb{R}^{q}$.

1. Solve $L_{B} t_{1}=r_{1}$ and $L_{C} t_{2}=r_{2}$ to get $t_{1}$ and $t_{2}$, and let $t_{2}:=t_{2}+$ $\bar{F} t_{1}$.

2. Solve $W w=t$ to get $w$, with $t=\left(t_{1}^{T}, t_{2}^{T}\right)^{T}$.

3. Solve $R_{C} z_{2}=w_{2}$ and $R_{B} z_{1}=w_{1}-\bar{E} w_{2}$ to get $z_{1}$ and $z_{2}$.

When the approximation matrix $W \in \mathbb{R}^{n \times n}$ to the matrix $\bar{W} \in \mathbb{R}^{n \times n}$ is specified, a concrete procedure for computing the generalized residual vector $z \in \mathbb{R}^{n}$ defined by (2.18) can be straightforwardly obtained from this procedure.

Usually, the matrix $W \in \mathbb{R}^{n \times n}$ may involve information about the matrices $J_{B}$, $J_{C}, \bar{E}$ and $\bar{F}$. Therefore, to solve the linear system $W w=t$ we may need to compute the vectors

$$
\begin{cases}\bar{w}_{1}=J_{B} w_{1}=L_{B}^{-1} B R_{B}^{-1} w_{1}, & \widetilde{w}_{1}=\bar{F} w_{1}=L_{C}^{-1} F R_{B}^{-1} w_{1}, \\ \bar{w}_{2}=J_{C} w_{2}=L_{C}^{-1} C R_{C}^{-1} w_{2}, & \widetilde{w}_{2}=\bar{E} w_{2}=L_{B}^{-1} E R_{C}^{-1} w_{2} .\end{cases}
$$


These vectors can be economically computed by the following formulas:

1. Solve $R_{B} t_{1}=w_{1}$.

2. Solve $L_{B} \bar{w}_{1}=B t_{1}, L_{C} \widetilde{w}_{1}=F t_{1}$.

3. Solve $R_{C} t_{2}=w_{2}$.

4. Solve $L_{C} \bar{w}_{2}=C t_{2}, L_{B} \widetilde{w}_{2}=E t_{2}$.

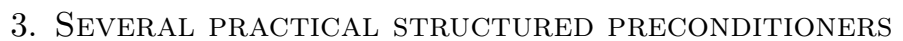

In this section, we will construct three classes of structured approximations $W$ to the block two-by-two matrix $\bar{W}$, or in other words, to the block two-by-two matrix $H$ in (2.7), by making use of the modified block Jacobi, the modified block Gauss-Seidel and the modified block unsymmetric Gauss-Seidel splittings of $\bar{W}$. See [6, 7] for details. Therefore, three types of structured preconditioners to the original block two-by-two matrix $A \in \mathbb{R}^{n \times n}$, called the modified block Jacobi-type (MBJ-type) preconditioner, the modified block Gauss-Seidel-type (MBGS-type) preconditioner and the modified block unsymmetric Gauss-Seidel-type (MBUGStype) preconditioner, can be obtained, correspondingly.

To analyze the spectral property of the preconditioned matrices with respect to the above-mentioned preconditioners, we need the following two basic facts.

Lemma 3.1. Let $L \in \mathbb{R}^{(p+q) \times(p+q)}$ and $U \in \mathbb{R}^{(p+q) \times(p+q)}$ be unit lower and upper triangular matrices of the block two-by-two forms

$$
L=\left[\begin{array}{cc}
I & O \\
L_{21} & I
\end{array}\right] \quad \text { and } \quad U=\left[\begin{array}{cc}
I & U_{12} \\
O & I
\end{array}\right]
$$

where $L_{21} \in \mathbb{R}^{q \times p}$ and $U_{12} \in \mathbb{R}^{p \times q}$. Let

$$
\gamma(t)=\left[1+\frac{1}{2} t\left(t+\sqrt{t^{2}+4}\right)\right]^{\frac{1}{2}}
$$

be a monotone increasing function with respect to $t$ in the interval $[0,+\infty)$. Then it follows that

$$
\|L\|_{2}=\gamma\left(\left\|L_{21}\right\|_{2}\right) \quad \text { and } \quad\|U\|_{2}=\gamma\left(\left\|U_{12}\right\|_{2}\right) .
$$

Proof. By direct computations we have

$$
L^{T} L=\left[\begin{array}{cc}
I & L_{21}^{T} \\
O & I
\end{array}\right]\left[\begin{array}{cc}
I & O \\
L_{21} & I
\end{array}\right]=\left[\begin{array}{cc}
I+L_{21}^{T} L_{21} & L_{21}^{T} \\
L_{21} & I
\end{array}\right]
$$

Without loss of generality, we assume $p \geq q$. From Theorem 2.5.2 in [27, page 70] we know that the matrix $L_{21}$ admits a singular value decomposition (SVD), i.e., there exist two orthogonal matrices $V_{1} \in \mathbb{R}^{q \times q}$ and $V_{2} \in \mathbb{R}^{p \times p}$ and a matrix $\widetilde{\Sigma}=$ $[\Sigma, O] \in \mathbb{R}^{q \times p}$, with $\Sigma=\operatorname{diag}\left(\sigma_{1}, \sigma_{2}, \ldots, \sigma_{q}\right) \in \mathbb{R}^{q \times q}$ being a nonnegative diagonal matrix having the maximum diagonal entry $\sigma_{1}=\left\|L_{21}\right\|_{2}$, such that $L_{21}=V_{1}^{T} \widetilde{\Sigma} V_{2}$ holds. Define

$$
V=\left[\begin{array}{cc}
V_{2} & O \\
O & V_{1}
\end{array}\right] .
$$

Then $V$ is an orthogonal matrix, too. It follows from concrete computations that

$$
L^{T} L=V^{T}\left[\begin{array}{ccc}
I+\Sigma^{2} & O & \Sigma \\
O & I & O \\
\Sigma & O & I
\end{array}\right] V .
$$


Therefore, detailed analysis shows that the eigenvalues of the matrix $L^{T} L$ are 1 with multiplicity $p-q$ and

$$
1+\frac{1}{2} \sigma_{k}\left(\sigma_{k} \pm \sqrt{\sigma_{k}^{2}+4}\right), \quad k=1,2, \ldots, q .
$$

It then follows straightforwardly that the spectral radius of the matrix $L^{T} L$, say $\rho\left(L^{T} L\right)$, is given by

$$
\begin{aligned}
\rho\left(L^{T} L\right) & =1+\frac{1}{2} \sigma_{1}\left(\sigma_{1}+\sqrt{\sigma_{1}^{2}+4}\right) \\
& =1+\frac{1}{2}\left\|L_{21}\right\|_{2}\left(\left\|L_{21}\right\|_{2}+\sqrt{\left\|L_{21}\right\|_{2}^{2}+4}\right),
\end{aligned}
$$

and therefore,

$$
\|L\|_{2}=\rho\left(L^{T} L\right)^{\frac{1}{2}}=\left[1+\frac{1}{2}\left\|L_{21}\right\|_{2}\left(\left\|L_{21}\right\|_{2}+\sqrt{\left\|L_{21}\right\|_{2}^{2}+4}\right)\right]^{\frac{1}{2}}=\gamma\left(\left\|L_{21}\right\|_{2}\right) .
$$

The proof of the second equality can be demonstrated in a similar fashion.

We remark that for the real one-variable function $\gamma(t)$ defined by (3.1), the estimate $\gamma(t) \leq t+1$ holds for all $t \in[0,+\infty)$ because of $\sqrt{t^{2}+4} \leq t+2$ and $\sqrt{t^{2}+t+1} \leq t+1$.

Lemma 3.2. Let $\Lambda=\operatorname{diag}\left(\lambda_{1}, \lambda_{2}, \ldots, \lambda_{n}\right) \in \mathbb{C}^{n \times n}$ be a diagonal matrix, and $Y \in \mathbb{C}^{n \times n}$ a given matrix, where $\mathbb{C}^{n \times n}$ represents the complex $n \times n$ matrix space. If there exists a positive constant $\rho_{y}$ such that $\|\Lambda-Y\|_{2} \leq \rho_{y}$, then all eigenvalues of the matrix $Y$ are located within $\bigcup_{i=1}^{n} \mathcal{N}\left(\lambda_{i}, \rho_{y}\right)$, where $\mathcal{N}\left(\lambda_{i}, \rho_{y}\right)$ denotes the circle having center $\lambda_{i}$ and radius $\rho_{y}$ on the complex plane.

Proof. Let $\lambda$ be an eigenvalue of the matrix $Y \in \mathbb{C}^{n \times n}$ and $v$ be the corresponding normalized eigenvector. Then we have $(\Lambda-Y) v=(\Lambda-\lambda I) v$. Hence,

$$
\|(\Lambda-\lambda I) v\|_{2}=\|(\Lambda-Y) v\|_{2} \leq\|\Lambda-Y\|_{2} \leq \rho_{y} .
$$

It then follows that $\|\Lambda-\lambda I\|_{2} \leq \rho_{y}$. Therefore, it follows that $\left|\lambda-\lambda_{i}\right| \leq \rho_{y}$ $(i=1,2, \ldots, n)$, or equivalently, $\lambda \in \bigcup_{i=1}^{n} \mathcal{N}\left(\lambda_{i}, \rho_{y}\right)$.

For the simplicity of our statements, in the sequel we always use $\gamma:(0,+\infty) \rightarrow$ $(0,+\infty)$ to represent the function defined by (3.1). For the matrices $J_{B}$ in (2.1) and $\bar{E}, \bar{F}$ in (2.3), we write

$$
\bar{\Delta}_{1}=\bar{F}\left(I-J_{B}\right) \bar{E} \text { and } \bar{\Delta}_{2}=\bar{F}\left(I-J_{B}\right)^{2} \bar{E},
$$

and denote the $(2,2)$-block entry of the matrix $\bar{W}$ in (2.7) by $\bar{S}$, i.e.,

$$
\bar{S}=J_{C}-\overline{F E} \text {. }
$$

Assume $\bar{W}$ and $\bar{S}$ are nonsingular, let $S$ be a nonsingular matrix that is a replacement to $\bar{S}$ (e.g., $S= \pm(I-\overline{F E})$ or $S= \pm(I-\operatorname{diag}(\overline{F E}))$, etc.), and define the quantities

$$
\Theta=\|\bar{E}\|_{2}, \quad \Gamma=\|\bar{F}\|_{2}, \quad \Theta_{s}=\left\|\bar{E} S^{-1}\right\|_{2}, \quad \Gamma_{s}=\left\|S^{-1} \bar{F}\right\|_{2} .
$$

In addition, in the case that $S$ is an approximation to $\bar{S}$, we define the quantities

$$
\epsilon_{L}=\max \left\{\left\|I-J_{B}\right\|_{2},\left\|I-S^{-1} \bar{S}\right\|_{2}\right\}, \quad \epsilon_{R}=\max \left\{\left\|I-J_{B}\right\|_{2},\left\|I-\bar{S} S^{-1}\right\|_{2}\right\} ;
$$


and in the case that $S$ is an approximation to $-\bar{S}$, instead of $\epsilon_{L}$ and $\epsilon_{R}$ we use the quantities

$$
\tilde{\epsilon}_{L}=\max \left\{\left\|I-J_{B}\right\|_{2},\left\|I+S^{-1} \bar{S}\right\|_{2}\right\}, \quad \widetilde{\epsilon}_{R}=\max \left\{\left\|I-J_{B}\right\|_{2},\left\|I+\bar{S} S^{-1}\right\|_{2}\right\} .
$$

For two positive constants $\rho_{L}^{(\xi)}$ and $\rho_{R}^{(\xi)}$, to be specified later, we use $\mathcal{N}^{(\xi)}$ to denote the circle having center $(1,0)$ and radius $\rho^{(\xi)}:=\min \left\{\rho_{L}^{(\xi)} \epsilon_{L}, \rho_{R}^{(\xi)} \epsilon_{R}\right\}$, and use $\tilde{\mathcal{N}}^{(\xi)}$ to denote the union of the two circles having centers $(-1,0)$ and $(1,0)$ and radius $\widetilde{\rho}^{(\xi)}:=\min \left\{\rho_{L}^{(\xi)} \widetilde{\epsilon}_{L}, \rho_{R}^{(\xi)} \widetilde{\epsilon}_{R}\right\}$, on the complex plane, respectively.

By making use of the above notation, the nonsingularity of the matrices $\bar{S}$ and $\bar{W}$ can be precisely described by the following lemma.

Lemma 3.3. The matrices $\bar{S}$ and $\bar{W}$ are nonsingular, provided either of the following conditions holds:

(1) $S$ is an approximation to $\bar{S}$, and

(a) $\epsilon_{L}<1+\Theta \Gamma_{s}-\sqrt{\Theta \Gamma_{s}\left(\Theta \Gamma_{s}+2\right)}$, or

(b) $\epsilon_{R}<1+\Theta_{s} \Gamma-\sqrt{\Theta_{s} \Gamma\left(\Theta_{s} \Gamma+2\right)}$;

(2) $S$ is an approximation to $-\bar{S}$, and

(a) $\tilde{\epsilon}_{L}<1+\Theta \Gamma_{s}-\sqrt{\Theta \Gamma_{s}\left(\Theta \Gamma_{s}+2\right)}$, or

(b) $\tilde{\epsilon}_{R}<1+\Theta_{s} \Gamma-\sqrt{\Theta_{s} \Gamma\left(\Theta_{s} \Gamma+2\right)}$.

Proof. We only prove (1a), as the other conclusions can be demonstrated analogously.

Because $\left\|I-J_{B}\right\|_{2} \leq \epsilon_{L}<1$, it follows that

$$
\left\|J_{B}^{-1}\right\|_{2}=\left\|\left[I-\left(I-J_{B}\right)\right]^{-1}\right\|_{2} \leq \frac{1}{1-\left\|I-J_{B}\right\|_{2}} .
$$

From (2.8) we have

$$
S_{\bar{A}}=\bar{S}-\bar{F}\left(I-J_{B}\right) J_{B}^{-1} \bar{E}
$$

Hence,

$$
\begin{aligned}
\left\|I-S^{-1} S_{\bar{A}}\right\|_{2} & \leq\left\|I-S^{-1} \bar{S}\right\|_{2}+\|\bar{E}\|_{2}\left\|S^{-1} \bar{F}\right\|_{2} \cdot \frac{\left\|I-J_{B}\right\|_{2}}{1-\left\|I-J_{B}\right\|_{2}} \\
& \leq\left(1+\frac{\Theta \Gamma_{s}}{1-\left\|I-J_{B}\right\|_{2}}\right) \cdot \max \left\{\left\|I-J_{B}\right\|_{2}, \quad\left\|I-S^{-1} \bar{S}\right\|_{2}\right\} \\
& \leq\left(1+\frac{\Theta \Gamma_{s}}{1-\epsilon_{L}}\right) \epsilon_{L} \\
& <1 .
\end{aligned}
$$

It then follows that

$$
\left\|S_{\bar{A}}^{-1} \bar{F}\right\|_{2} \leq \frac{\left\|S^{-1} \bar{F}\right\|_{2}}{1-\left\|I-S^{-1} S_{\bar{A}}\right\|_{2}} \leq \frac{\Gamma_{s}\left(1-\epsilon_{L}\right)}{\left(1-\epsilon_{L}\right)^{2}-\Theta \Gamma_{s} \epsilon_{L}} .
$$

Now, we easily see that (2.9) holds when

$$
\epsilon_{L}<\frac{1}{1+\frac{\Theta \Gamma_{s}\left(1-\epsilon_{L}\right)}{\left(1-\epsilon_{L}\right)^{2}-\Theta \Gamma_{s} \epsilon_{L}}}=\frac{\left(1-\epsilon_{L}\right)^{2}-\Theta \Gamma_{s} \epsilon_{L}}{\left(1-\epsilon_{L}\right)^{2}-\Theta \Gamma_{s} \epsilon_{L}+\Theta \Gamma_{s}\left(1-\epsilon_{L}\right)}
$$


or equivalently, $\epsilon_{L}^{2}-2\left(1+\Theta \Gamma_{s}\right) \epsilon_{L}+1>0$. Therefore, when

$$
\epsilon_{L}<1+\Theta \Gamma_{s}-\sqrt{\Theta \Gamma_{s}\left(\Theta \Gamma_{s}+2\right)},
$$

the matrices $\bar{S}$ and $\bar{W}$ are nonsingular.

We first consider the case that $S \approx \bar{S}$. The case that $S \approx-\bar{S}$ will be discussed in Section 3.4

3.1. The MBJ-type preconditioners. If the matrix $W \in \mathbb{R}^{n \times n}$ in (2.10) is taken to be the modified block Jacobi splitting matrix [6, 7] of the matrix $\bar{W}$ in (2.7), i.e.,

$$
W^{(J)}:=W=\left[\begin{array}{cc}
I & O \\
O & S
\end{array}\right],
$$

then we obtain the modified block Jacobi-type (MBJ-type) preconditioner $M=$ $P W^{(J)} Q$ to the original matrix $A \in \mathbb{R}^{n \times n}$. Note that when $S \in \mathbb{R}^{q \times q}$ is symmetric positive definite, $W^{(J)}$ is a symmetric positive definite matrix, and when $S \in \mathbb{R}^{q \times q}$ is symmetric negative definite, $W^{(J)}$ is a symmetric indefinite matrix.

The following theorem describes the eigenvalue distribution of the preconditioned matrix with respect to the MBJ-type preconditioner.

Theorem 3.1. Let $M=P W^{(J)} Q$ be the $M B J$-type preconditioner to the block two-by-two matrix $A=P H Q \in \mathbb{R}^{n \times n}$ in (1.2), where $P$ and $Q$ are given by (2.6), $H$ is given by (2.7), and $W^{(J)}$ is defined by (3.3). Let $K_{L}=W^{(J)^{-1}} H$ and $K_{R}=$ $H W^{(J)^{-1}}$. Then it follows that

(i) $\left\|I-K_{L}\right\|_{2} \leq \rho_{L}^{(J)} \epsilon_{L}$, with $\rho_{L}^{(J)}=\gamma(\Theta) \cdot \gamma\left(\Gamma_{s}\right)$; and

(ii) $\left\|I-K_{R}\right\|_{2} \leq \rho_{R}^{(J)} \epsilon_{R}$, with $\rho_{R}^{(J)}=\gamma\left(\Theta_{s}\right) \cdot \gamma(\Gamma)$.

It follows from Lemma 3.2 as well as (2.11) and (2.13) that the eigenvalues of the matrices $M^{-1} A$ and $A M^{-1}$ are located within a circle having center $(1,0)$ and radii $\rho_{L}^{(J)} \epsilon_{L}$ and $\rho_{R}^{(J)} \epsilon_{R}$, respectively, and therefore, they are all within the circle $\mathcal{N}^{(J)}$.

Proof. We only prove (i), as (ii) can be verified analogously.

From (2.7) and (3.3) we have

$$
K_{L}=W^{(J)^{-1}} H=\left[\begin{array}{cc}
J_{B} & \left(I-J_{B}\right) \bar{E} \\
S^{-1} \bar{F}\left(I-J_{B}\right) & S^{-1} \bar{S}-S^{-1} \bar{\Delta}_{1}
\end{array}\right] .
$$

Hence,

$$
I-K_{L}=\left[\begin{array}{cc}
I & O \\
-S^{-1} \bar{F} & I
\end{array}\right]\left[\begin{array}{cc}
I-J_{B} & O \\
O & I-S^{-1} \bar{S}
\end{array}\right]\left[\begin{array}{cc}
I & -\bar{E} \\
O & I
\end{array}\right] .
$$

By making use of Lemma 3.1 we can immediately obtain

$$
\begin{aligned}
\left\|I-K_{L}\right\|_{2} & \leq \gamma\left(\left\|S^{-1} \bar{F}\right\|_{2}\right) \cdot \gamma\left(\|\bar{E}\|_{2}\right) \cdot \max \left\{\left\|I-J_{B}\right\|_{2}, \quad\left\|I-S^{-1} \bar{S}\right\|_{2}\right\} \\
& =\gamma(\Theta) \cdot \gamma\left(\Gamma_{s}\right) \cdot \max \left\{\left\|I-J_{B}\right\|_{2}, \quad\left\|I-S^{-1} \bar{S}\right\|_{2}\right\} \\
& =\rho_{L}^{(J)} \epsilon_{L} .
\end{aligned}
$$

Furthermore, when the matrix $J_{B}$ is positive definite, we can demonstrate the positive definiteness of the matrices $K_{L}=W^{(J)^{-1}} H$ and $K_{R}=H W^{(J)^{-1}}$. 
Theorem 3.2. Let the matrix $J_{B}$ be positive definite. Then

(i) the matrix $K_{L}=W^{(J)^{-1}} H$ is positive definite, provided $\epsilon_{L}<\delta_{L}^{(J)}$, where

$$
\delta_{L}^{(J)}=\frac{2\left(\Theta \Gamma_{s}+2-\sqrt{\Theta^{2} \Gamma_{s}^{2}+\left(\Theta+\Gamma_{s}\right)^{2}}\right)}{4-\left(\Theta-\Gamma_{s}\right)^{2}}<1 ;
$$

(ii) the matrix $K_{R}=H W^{(J)^{-1}}$ is positive definite, provided $\epsilon_{R}<\delta_{R}^{(J)}$, where

$$
\delta_{R}^{(J)}=\frac{2\left(\Theta_{s} \Gamma+2-\sqrt{\Theta_{s}^{2} \Gamma^{2}+\left(\Theta_{s}+\Gamma\right)^{2}}\right)}{4-\left(\Theta_{s}-\Gamma\right)^{2}}<1 .
$$

Proof. We only prove the validity of (i), as (ii) can be demonstrated similarly.

Some straightforward computations immediately show that $\delta_{L}^{(J)}<1$. Let

$$
T=\left[\begin{array}{ll}
T_{11} & T_{12} \\
T_{21} & T_{22}
\end{array}\right] \equiv \frac{1}{2}\left(K_{L}+K_{L}^{T}\right) .
$$

Then from the proof of Theorem 3.1 we easily obtain

$$
\left\{\begin{array}{l}
T_{11}=\frac{1}{2}\left(J_{B}+J_{B}^{T}\right), \\
T_{12}=\frac{1}{2}\left[\left(I-J_{B}\right) \bar{E}+\left(I-J_{B}^{T}\right) \bar{F}^{T} S^{-T}\right], \\
T_{21}=\frac{1}{2}\left[S^{-1} \bar{F}\left(I-J_{B}\right)+\bar{E}^{T}\left(I-J_{B}^{T}\right)\right] \\
T_{22}=\frac{1}{2}\left[S^{-1} \bar{S}+\bar{S}^{T} S^{-T}\right]-\frac{1}{2}\left[S^{-1} \bar{\Delta}_{1}+\bar{\Delta}_{1}^{T} S^{-T}\right] .
\end{array}\right.
$$

Because $J_{B}$ is positive definite, we know that its symmetric part $\frac{1}{2}\left(J_{B}+J_{B}^{T}\right)$ is symmetric positive definite. Therefore, the matrix $T$ is symmetric positive definite if and only if so is its Schur complement $S_{T}:=T_{22}-T_{21} T_{11}^{-1} T_{12}$.

Since

$$
\left\|I-T_{11}\right\|_{2} \leq\left\|I-J_{B}\right\|_{2} \leq \epsilon_{L}<\delta_{L}^{(J)}<1,
$$

we have

$$
\left\|T_{11}^{-1}\right\|_{2}=\left\|\left[I-\left(I-T_{11}\right)\right]^{-1}\right\|_{2} \leq \frac{1}{1-\left\|I-J_{B}\right\|_{2}} .
$$

By direct computations we immediately obtain

$$
\begin{aligned}
\left\|T_{12}\right\|_{2}=\left\|T_{21}\right\|_{2} & \leq \frac{1}{2}\left(\left\|S^{-1} \bar{F}\right\|_{2}\left\|I-J_{B}\right\|_{2}+\left\|\bar{E}^{T}\right\|_{2}\left\|I-J_{B}^{T}\right\|_{2}\right) \\
& =\frac{1}{2}\left(\Theta+\Gamma_{s}\right)\left\|I-J_{B}\right\|_{2}
\end{aligned}
$$

and

$$
\begin{aligned}
\left\|I-T_{22}\right\|_{2} \leq & \frac{1}{2}\left(\left\|I-S^{-1} \bar{S}\right\|_{2}+\left\|I-\bar{S}^{T} S^{-T}\right\|_{2}\right) \\
& +\frac{1}{2}\left(\left\|S^{-1} \bar{\Delta}_{1}\right\|_{2}+\left\|\bar{\Delta}_{1}^{T} S^{-T}\right\|_{2}\right) \\
\leq & \left\|I-S^{-1} \bar{S}\right\|_{2}+\left\|S^{-1} \bar{F}\right\|_{2}\|\bar{E}\|_{2}\left\|I-J_{B}\right\|_{2} \\
\leq & \left(1+\Theta \Gamma_{s}\right) \cdot \max \left\{\left\|I-J_{B}\right\|_{2}, \quad\left\|I-S^{-1} \bar{S}\right\|_{2}\right\} \\
= & \left(1+\Theta \Gamma_{s}\right) \epsilon_{L} .
\end{aligned}
$$


It then follows that

$$
\begin{aligned}
\min _{x \neq 0} \frac{\left\langle x, S_{T} x\right\rangle}{\langle x, x\rangle} & \geq 1-\max _{x \neq 0} \frac{\left\langle x,\left(I-T_{22}\right) x\right\rangle}{\langle x, x\rangle}-\max _{x \neq 0} \frac{\left\langle x, T_{21} T_{11}^{-1} T_{12} x\right\rangle}{\langle x, x\rangle} \\
& \geq 1-\left\|I-T_{22}\right\|_{2}-\left\|T_{21} T_{11}^{-1} T_{12}\right\|_{2} \\
& \geq 1-\left(\left(1+\Theta \Gamma_{s}\right) \epsilon_{L}+\frac{\left(\Theta+\Gamma_{s}\right)^{2}\left\|I-J_{B}\right\|_{2}^{2}}{4\left(1-\left\|I-J_{B}\right\|_{2}\right)}\right) \\
& \geq 1-\left(1+\Theta \Gamma_{s}+\frac{\left(\Theta+\Gamma_{s}\right)^{2}}{4\left(1-\left\|I-J_{B}\right\|_{2}\right)} \cdot\left\|I-J_{B}\right\|_{2}\right) \epsilon_{L} \\
& \geq 1-\left(1+\Theta \Gamma_{s}+\frac{\left(\Theta+\Gamma_{s}\right)^{2}}{4\left(1-\epsilon_{L}\right)} \cdot \epsilon_{L}\right) \epsilon_{L} .
\end{aligned}
$$

Noticing that

$$
\left(1+\Theta \Gamma_{s}+\frac{\left(\Theta+\Gamma_{s}\right)^{2}}{4\left(1-\epsilon_{L}\right)} \cdot \epsilon_{L}\right) \epsilon_{L}<1
$$

holds if and only if

$$
4 \Theta \Gamma_{s} \epsilon_{L}+\left(\Theta-\Gamma_{s}\right)^{2} \epsilon_{L}^{2}<4\left(1-\epsilon_{L}\right)^{2},
$$

or equivalently,

$$
\epsilon_{L}<\frac{2\left(\Theta \Gamma_{s}+2-\sqrt{\Theta^{2} \Gamma_{s}^{2}+\left(\Theta+\Gamma_{s}\right)^{2}}\right)}{4-\left(\Theta-\Gamma_{s}\right)^{2}},
$$

we therefore know that $\min _{x \neq 0} \frac{\left\langle x, S_{T} x\right\rangle}{\langle x, x\rangle}>0$ holds true when $\epsilon_{L}<\delta_{L}^{(J)}$. Hence, $S_{T}$ is a symmetric positive definite matrix, and $K_{L}$ is a positive definite matrix.

3.2. The MBGS-type preconditioners. If the matrix $W \in \mathbb{R}^{n \times n}$ in (2.10) is taken to be the modified block Gauss-Seidel splitting matrix [6, 7] of the matrix $\bar{W}$ in (2.7), i.e.,

$$
W^{(G S)}:=W=\left[\begin{array}{cc}
I & O \\
\bar{F}\left(I-J_{B}\right) & S
\end{array}\right],
$$

then we obtain the modified block Gauss-Seidel-type (MBGS-type) preconditioner $M=P W^{(G S)} Q$ to the original matrix $A \in \mathbb{R}^{n \times n}$.

The following theorem describes the eigenvalue distribution of the preconditioned matrix with respect to the MBGS-type preconditioner.

Theorem 3.3. Let $M=P W^{(G S)} Q$ be the $M B G S$-type preconditioner to the block two-by-two matrix $A=P H Q \in \mathbb{R}^{n \times n}$ in (1.2), where $P$ and $Q$ are given by (2.6), $H$ is given by (2.7), and $W^{(G S)}$ is defined by (3.4). Let $K_{L}=W^{(G S)^{-1}} H$ and $K_{R}=H W^{(G S)^{-1}}$. Then it follows that

(i) $\left\|I-K_{L}\right\|_{2} \leq \rho_{L}^{(G S)} \epsilon_{L}$, with $\rho_{L}^{(G S)}=\gamma(\Theta) \cdot \gamma\left(\Gamma_{s}\left\|I-J_{B}\right\|_{2}\right) \cdot\left(1+\Theta \Gamma_{s}\right)$; and

(ii) $\left\|I-K_{R}\right\|_{2} \leq \rho_{R}^{(G S)} \epsilon_{R}$, with $\rho_{R}^{(G S)}=\gamma\left(\Theta_{s}\right) \cdot \gamma\left(\Gamma\left\|I-J_{B}\right\|_{2}\right) \cdot\left(1+\Theta_{s} \Gamma\right)$.

It follows from Lemma 3.2 as well as (2.11) and (2.13) that the eigenvalues of the matrices $M^{-1} A$ and $A M^{-1}$ are located within a circle having center $(1,0)$ and radii $\rho_{L}^{(G S)} \epsilon_{L}$ and $\rho_{R}^{(G S)} \epsilon_{R}$, respectively, and therefore, they are all within the circle $\mathcal{N}^{(G S)}$. 
Proof. We only prove (i), as (ii) can be verified analogously.

From (2.7) and (3.4) we have

$$
K_{L}=W^{(G S)^{-1}} H=\left[\begin{array}{cc}
J_{B} & \left(I-J_{B}\right) \bar{E} \\
S^{-1} \bar{F}\left(I-J_{B}\right)^{2} & S^{-1} \bar{S}-S^{-1}\left(\bar{\Delta}_{1}+\bar{\Delta}_{2}\right)
\end{array}\right] .
$$

Hence,

$$
I-K_{L}=\left[\begin{array}{cc}
I & O \\
-S^{-1} \bar{F}\left(I-J_{B}\right) & I
\end{array}\right]\left[\begin{array}{cc}
I-J_{B} & O \\
O & I-S^{-1} \bar{S}+S^{-1} \bar{\Delta}_{1}
\end{array}\right]\left[\begin{array}{cc}
I & -\bar{E} \\
O & I
\end{array}\right] .
$$

By making use of Lemma 3.1 we can immediately obtain

$$
\begin{aligned}
\left\|I-K_{L}\right\|_{2} \leq & \gamma\left(\left\|S^{-1} \bar{F}\left(I-J_{B}\right)\right\|_{2}\right) \cdot \gamma\left(\|\bar{E}\|_{2}\right) \\
& \cdot \max \left\{\left\|I-J_{B}\right\|_{2}, \quad\left\|I-S^{-1} \bar{S}\right\|_{2}+\left\|S^{-1} \bar{F}\right\|_{2}\|\bar{E}\|_{2}\left\|I-J_{B}\right\|_{2}\right\} \\
\leq & \gamma(\Theta) \cdot \gamma\left(\Gamma_{s}\left\|I-J_{B}\right\|_{2}\right) \cdot\left(1+\Theta \Gamma_{s}\right) \\
& \quad \cdot \max \left\{\left\|I-J_{B}\right\|_{2}, \quad\left\|I-S^{-1} \bar{S}\right\|_{2}\right\} \\
= & \rho_{L}^{(G S)} \epsilon_{L} .
\end{aligned}
$$

Furthermore, when the matrix $J_{B}$ is positive definite, we can demonstrate the positive definiteness of the matrices $K_{L}=W^{(G S)^{-1}} H$ and $K_{R}=H W^{(G S)^{-1}}$.

Theorem 3.4. Let the matrix $J_{B}$ be positive definite. Then

(i) the matrix $K_{L}=W^{(G S)^{-1}} H$ is positive definite, provided $\epsilon_{L}<\delta_{L}^{(G S)}$, where

$$
\delta_{L}^{(G S)}=\frac{2\left(\Theta \Gamma_{s}+2-\sqrt{\Theta^{2} \Gamma_{s}^{2}+4 \Theta \Gamma_{s}+\left(\Theta+\Gamma_{s}\right)^{2}}\right)}{4-\left(\Theta+\Gamma_{s}\right)^{2}}<1 ;
$$

(ii) the matrix $K_{R}=H W^{(G S)^{-1}}$ is positive definite, provided $\epsilon_{R}<\delta_{R}^{(G S)}$, where

$$
\delta_{R}^{(G S)}=\frac{2\left(\Theta_{s} \Gamma+2-\sqrt{\Theta_{s}^{2} \Gamma^{2}+4 \Theta_{s} \Gamma+\left(\Theta_{s}+\left(1+\Theta_{s} \Gamma\right) \Gamma\right)^{2}}\right)}{4-\left(\Theta_{s}+\left(1+\Theta_{s} \Gamma\right) \Gamma\right)^{2}}<1 .
$$

Proof. We only prove the validity of (i), as (ii) can be demonstrated similarly.

Some straightforward computations immediately show that $\delta_{L}^{(G S)}<1$. Let

$$
T=\left[\begin{array}{ll}
T_{11} & T_{12} \\
T_{21} & T_{22}
\end{array}\right] \equiv \frac{1}{2}\left(K_{L}+K_{L}^{T}\right) .
$$

Then from the proof of Theorem 3.3 we can easily obtain

$$
\left\{\begin{array}{l}
T_{11}=\frac{1}{2}\left(J_{B}+J_{B}^{T}\right), \\
T_{12}=\frac{1}{2}\left[\left(I-J_{B}\right) \bar{E}+\left(I-J_{B}^{T}\right)^{2} \bar{F}^{T} S^{-T}\right] \\
T_{21}=\frac{1}{2}\left[S^{-1} \bar{F}\left(I-J_{B}\right)^{2}+\bar{E}^{T}\left(I-J_{B}^{T}\right)\right] \\
T_{22}=\frac{1}{2}\left[S^{-1} \bar{S}+\bar{S}^{T} S^{-T}\right]-\frac{1}{2}\left[S^{-1} \bar{\Delta}_{1}+\bar{\Delta}_{1}^{T} S^{-T}\right]-\frac{1}{2}\left[S^{-1} \bar{\Delta}_{2}+\bar{\Delta}_{2}^{T} S^{-T}\right] .
\end{array}\right.
$$

Because $J_{B}$ is positive definite, we know that its symmetric part $\frac{1}{2}\left(J_{B}+J_{B}^{T}\right)$ is symmetric positive definite. Therefore, the matrix $T$ is symmetric positive definite if and only if so is its Schur complement $S_{T}:=T_{22}-T_{21} T_{11}^{-1} T_{12}$. 
Since

$$
\left\|I-T_{11}\right\|_{2} \leq\left\|I-J_{B}\right\|_{2} \leq \epsilon_{L}<\delta_{L}^{(G S)}<1
$$

we have

$$
\left\|T_{11}^{-1}\right\|_{2}=\left\|\left[I-\left(I-T_{11}\right)\right]^{-1}\right\|_{2} \leq \frac{1}{1-\left\|I-J_{B}\right\|_{2}} .
$$

By direct computations we immediately get

$$
\begin{aligned}
\left\|T_{12}\right\|_{2}=\left\|T_{21}\right\|_{2} & \leq \frac{1}{2}\left(\left\|S^{-1} \bar{F}\left(I-J_{B}\right)^{2}\right\|_{2}+\left\|\bar{E}^{T}\left(I-J_{B}^{T}\right)\right\|_{2}\right) \\
& \leq \frac{1}{2}\left(\Theta+\Gamma_{s}\left\|I-J_{B}\right\|_{2}\right)\left\|I-J_{B}\right\|_{2}
\end{aligned}
$$

and

$$
\begin{aligned}
\left\|I-T_{22}\right\|_{2} \leq & \frac{1}{2}\left(\left\|I-S^{-1} \bar{S}\right\|_{2}+\left\|I-\bar{S}^{T} S^{-T}\right\|_{2}\right) \\
& +\frac{1}{2}\left(\left\|S^{-1} \bar{F}\left(I-J_{B}\right) \bar{E}\right\|_{2}+\left\|\bar{E}^{T}\left(I-J_{B}^{T}\right) \bar{F}^{T} S^{-T}\right\|_{2}\right) \\
& +\frac{1}{2}\left(\left\|S^{-1} \bar{F}\left(I-J_{B}\right)^{2} \bar{E}\right\|_{2}+\left\|\bar{E}^{T}\left(I-J_{B}^{T}\right)^{2} \bar{F}^{T} S^{-T}\right\|_{2}\right) \\
\leq & \left\|I-S^{-1} \bar{S}\right\|_{2}+\Theta \Gamma_{s}\left(1+\left\|I-J_{B}\right\|_{2}\right)\left\|I-J_{B}\right\|_{2} \\
\leq & {\left[1+\Theta \Gamma_{s}\left(1+\left\|I-J_{B}\right\|_{2}\right)\right] \cdot \max \left\{\left\|I-J_{B}\right\|_{2}, \quad\left\|I-S^{-1} \bar{S}\right\|_{2}\right\} } \\
\leq & {\left[1+\Theta \Gamma_{s}\left(1+\epsilon_{L}\right)\right] \epsilon_{L} . }
\end{aligned}
$$

It then follows that

$$
\begin{aligned}
\min _{x \neq 0} \frac{\left\langle x, S_{T} x\right\rangle}{\langle x, x\rangle} & \geq 1-\max _{x \neq 0} \frac{\left\langle x,\left(I-T_{22}\right) x\right\rangle}{\langle x, x\rangle}-\max _{x \neq 0} \frac{\left\langle x, T_{21} T_{11}^{-1} T_{12} x\right\rangle}{\langle x, x\rangle} \\
& \geq 1-\left\|I-T_{22}\right\|_{2}-\left\|T_{21} T_{11}^{-1} T_{12}\right\|_{2} \\
& \geq 1-\left[1+\Theta \Gamma_{s}\left(1+\epsilon_{L}\right)\right] \epsilon_{L}-\frac{\left(\Theta+\Gamma_{s}\left\|I-J_{B}\right\|_{2}\right)^{2}\left\|I-J_{B}\right\|_{2}^{2}}{4\left(1-\left\|I-J_{B}\right\|_{2}\right)} \\
& \geq 1-\left(1+\Theta \Gamma_{s}\left(1+\epsilon_{L}\right)+\frac{\left(\Theta+\Gamma_{s} \epsilon_{L}\right)^{2}}{4\left(1-\epsilon_{L}\right)} \cdot \epsilon_{L}\right) \epsilon_{L} .
\end{aligned}
$$

Notice that

$$
\left(1+\Theta \Gamma_{s}\left(1+\epsilon_{L}\right)+\frac{\left(\Theta+\Gamma_{s} \epsilon_{L}\right)^{2}}{4\left(1-\epsilon_{L}\right)} \cdot \epsilon_{L}\right) \epsilon_{L}<1
$$

holds if and only if

$$
4 \Theta \Gamma_{s}\left(1-\epsilon_{L}^{2}\right) \epsilon_{L}+\left(\Theta+\Gamma_{s} \epsilon_{L}\right)^{2} \epsilon_{L}^{2}<4\left(1-\epsilon_{L}\right)^{2},
$$

and this inequality holds when

$$
4 \Theta \Gamma_{s} \epsilon_{L}+\left(\Theta+\Gamma_{s}\right)^{2} \epsilon_{L}^{2}<4\left(1-\epsilon_{L}\right)^{2},
$$

or equivalently,

$$
\epsilon_{L}<\frac{2\left(\Theta \Gamma_{s}+2-\sqrt{\Theta^{2} \Gamma_{s}^{2}+4 \Theta \Gamma_{s}+\left(\Theta+\Gamma_{s}\right)^{2}}\right)}{4-\left(\Theta+\Gamma_{s}\right)^{2}} .
$$

Therefore, we know that $\min _{x \neq 0} \frac{\left\langle x, S_{T} x\right\rangle}{\langle x, x\rangle}>0$ holds true when $\epsilon_{L}<\delta_{L}^{(G S)}$. Hence, $S_{T}$ is a symmetric positive definite matrix, and $K_{L}$ is a positive definite matrix. 
Alternatively, if the matrix $W \in \mathbb{R}^{n \times n}$ in (2.10) is taken to be the modified block Gauss-Seidel splitting matrix [6, 7] of the matrix $\bar{W}$ in (2.7), i.e.,

$$
W^{(G S)}:=W=\left[\begin{array}{cc}
I & \left(I-J_{B}\right) \bar{E} \\
O & S
\end{array}\right],
$$

then we obtain another modified block Gauss-Seidel-type (MBGS-type) preconditioner $M=P W^{(G S)} Q$ to the original matrix $A \in \mathbb{R}^{n \times n}$. Exactly following the demonstrations of Theorems 3.3 and 3.4, we can obtain the following results for the eigenvalue distribution and the positive definiteness of the preconditioned matrix with respect to the MBGS-type preconditioner (3.5).

Theorem 3.5. Let $M=P W^{(G S)} Q$ be the $M B G S$-type preconditioner to the block two-by-two matrix $A=P H Q \in \mathbb{R}^{n \times n}$ in (1.2), where $P$ and $Q$ are given by (2.6), $H$ is given by (2.7), and $W^{(G S)}$ is defined by (3.5). Let $K_{L}=W^{(G S)^{-1}} H$ and $K_{R}=H W^{(G S)^{-1}}$. Then it follows that

(i) $\left\|I-K_{L}\right\|_{2} \leq \rho_{L}^{(G S)} \epsilon_{L}$, with $\rho_{L}^{(G S)}=\gamma\left(\Theta\left\|I-J_{B}\right\|_{2}\right) \cdot \gamma\left(\Gamma_{s}\right) \cdot\left(1+\Theta \Gamma_{s}\right)$; and

(ii) $\left\|I-K_{R}\right\|_{2} \leq \rho_{R}^{(G S)} \epsilon_{R}$, with $\rho_{R}^{(G S)}=\gamma\left(\Theta_{s}\left\|I-J_{B}\right\|_{2}\right) \cdot \gamma(\Gamma) \cdot\left(1+\Theta_{s} \Gamma\right)$.

It follows from Lemma 3.2 as well as (2.11) and (2.13) that the eigenvalues of the matrices $M^{-1} A$ and $A M^{-1}$ are located within a circle having center $(1,0)$ and radii $\rho_{L}^{(G S)} \epsilon_{L}$ and $\rho_{R}^{(G S)} \epsilon_{R}$, respectively, and therefore, they are all within the circle $\mathcal{N}^{(G S)}$.

Theorem 3.6. Let the matrix $J_{B}$ be positive definite. Then

(i) the matrix $K_{L}=W^{(G S)^{-1}} H$ is positive definite, provided $\epsilon_{L}<\delta_{L}^{(G S)}$, where

$$
\delta_{L}^{(G S)}=\frac{2\left(\Theta \Gamma_{s}+2-\sqrt{\Theta^{2} \Gamma_{s}^{2}+4 \Theta \Gamma_{s}+\left(\Gamma_{s}+\left(1+\Theta \Gamma_{s}\right) \Theta\right)^{2}}\right)}{4-\left(\Gamma_{s}+\left(1+\Theta \Gamma_{s}\right) \Theta\right)^{2}}<1
$$

(ii) the matrix $K_{R}=H W^{(G S)^{-1}}$ is positive definite, provided $\epsilon_{R}<\delta_{R}^{(G S)}$, where

$$
\delta_{R}^{(G S)}=\frac{2\left(\Theta_{s} \Gamma+2-\sqrt{\Theta_{s}^{2} \Gamma^{2}+4 \Theta_{s} \Gamma+\left(\Theta_{s}+\Gamma\right)^{2}}\right)}{4-\left(\Theta_{s}+\Gamma\right)^{2}}<1 .
$$

3.3. The MBUGS-type preconditioners. If the matrix $W \in \mathbb{R}^{n \times n}$ in (2.10) is taken to be the modified block unsymmetric Gauss-Seidel splitting matrix [6, 7] of the matrix $\bar{W}$ in (2.7), i.e.,

$$
W^{(U G S)}:=W=\left[\begin{array}{cc}
I & \left(I-J_{B}\right) \bar{E} \\
O & S
\end{array}\right]\left[\begin{array}{cc}
I & O \\
O & S
\end{array}\right]^{-1}\left[\begin{array}{cc}
I & O \\
\bar{F}\left(I-J_{B}\right) & S
\end{array}\right],
$$

then we obtain the modified block unsymmetric Gauss-Seidel-type (MBUGS-type) preconditioner $M=P W^{(U G S)} Q$ to the original matrix $A \in \mathbb{R}^{n \times n}$.

The following theorem describes the eigenvalue distribution of the preconditioned matrix with respect to the MBUGS-type preconditioner.

Theorem 3.7. Let $M=P W^{(U G S)} Q$ be the MBUGS-type preconditioner to the block two-by-two matrix $A=P H Q \in \mathbb{R}^{n \times n}$ in (1.2), where $P$ and $Q$ are given by (2.6), $H$ is given by (2.7), and $W^{(U G S)}$ is defined by (3.6). Let $K_{L}=W^{(U G S)^{-1}} H$ and $K_{R}=H W^{(U G S)^{-1}}$. Then it follows that 
(i) $\left\|I-K_{L}\right\|_{2} \leq \rho_{L}^{(U G S)} \epsilon_{L}$, with

$$
\rho_{L}^{(U G S)}=\gamma\left(\Gamma_{s}\left\|I-J_{B}\right\|_{2}\right) \cdot\left[\gamma\left(\Theta\left\|I-J_{B}\right\|_{2}\right)+\Theta \Gamma_{s}\left\|I-J_{B}\right\|_{2}\right] \cdot\left(1+\Theta \Gamma_{s}\right) ; \text { and }
$$

(ii) $\left\|I-K_{R}\right\|_{2} \leq \rho_{R}^{(U G S)} \epsilon_{R}$, with

$$
\rho_{R}^{(U G S)}=\gamma\left(\Theta_{s}\left\|I-J_{B}\right\|_{2}\right) \cdot\left[\gamma\left(\Gamma\left\|I-J_{B}\right\|_{2}\right)+\Theta_{s} \Gamma\left\|I-J_{B}\right\|_{2}\right] \cdot\left(1+\Theta_{s} \Gamma\right) .
$$

It follows from Lemma 3.2 as well as (2.11) and (2.13) that the eigenvalues of the matrices $M^{-1} A$ and $A M^{-1}$ are located within a circle having center $(1,0)$ and radii $\rho_{L}^{(U G S)} \epsilon_{L}$ and $\rho_{R}^{(U G S)} \epsilon_{R}$, respectively, and therefore, they are all within the circle $\mathcal{N}^{(U G S)}$.

Proof. It is analogous to the proofs of Theorems 3.1 and 3.3 and hence is omitted.

Furthermore, when the matrix $J_{B}$ is positive definite, we can demonstrate the positive definiteness of the matrices $K_{L}=W^{(U G S)^{-1}} H$ and $K_{R}=H W^{(U G S)^{-1}}$.

Theorem 3.8. Let the matrix $J_{B}$ be positive definite. Then

(i) the matrix $K_{L}=W^{(U G S)^{-1}} H$ is positive definite, provided $\epsilon_{L}<\delta_{L}^{(U G S)}$, where

$$
\delta_{L}^{(U G S)}=\frac{2\left(\Theta \Gamma_{s}+2-\sqrt{\Theta^{2} \Gamma_{s}^{2}+4 \Theta \Gamma_{s}+\left(1+\Theta \Gamma_{s}\right)^{2}\left(\Theta+\Gamma_{s}\right)^{2}}\right)}{4-\left(1+\Theta \Gamma_{s}\right)^{2}\left(\Theta+\Gamma_{s}\right)^{2}}<1 ;
$$

(ii) the matrix $K_{R}=H W^{(U G S)^{-1}}$ is positive definite, provided $\epsilon_{R}<\delta_{R}^{(U G S)}$, where

$$
\delta_{R}^{(U G S)}=\frac{2\left(\Theta_{s} \Gamma+2-\sqrt{\Theta_{s}^{2} \Gamma^{2}+4 \Theta_{s} \Gamma+\left(1+\Theta_{s} \Gamma\right)^{2}\left(\Theta_{s}+\Gamma\right)^{2}}\right)}{4-\left(1+\Theta_{s} \Gamma\right)^{2}\left(\Theta_{s}+\Gamma\right)^{2}}<1 .
$$

Proof. It is analogous to the proofs of Theorems 3.2 and 3.4 and hence is omitted.

Alternatively, if the matrix $W^{(U G S)}$ defined by (3.6) is considered to possess the split form $W^{(U G S)}=W_{L}^{(U G S)} W_{R}^{(U G S)}$, with

$$
W_{L}^{(U G S)}=\left[\begin{array}{cc}
I & \left(I-J_{B}\right) \bar{E} S^{-1} \\
O & I
\end{array}\right], \quad W_{R}^{(U G S)}=\left[\begin{array}{cc}
I & O \\
\bar{F}\left(I-J_{B}\right) & S
\end{array}\right]
$$

or

$$
W_{L}^{(U G S)}=\left[\begin{array}{cc}
I & \left(I-J_{B}\right) \bar{E} \\
O & S
\end{array}\right], \quad W_{R}^{(U G S)}=\left[\begin{array}{cc}
I & O \\
S^{-1} \bar{F}\left(I-J_{B}\right) & I
\end{array}\right],
$$

then we can obtain other modified block unsymmetric Gauss-Seidel-type preconditioners $M=M_{L}^{(U G S)} M_{R}^{(U G S)}$ to the original matrix $A \in \mathbb{R}^{n \times n}$, where

$$
M_{L}^{(U G S)}=P W_{L}^{(U G S)} \text { and } M_{R}^{(U G S)}=W_{R}^{(U G S)} Q,
$$

and $P$ and $Q$ are given by (2.6). Exactly following the demonstrations of Theorems 3.7 and 3.8. we can obtain the results about the eigenvalue distributions and the positive definiteness of the preconditioned matrices with respect to the MBUGS-type preconditioners (3.7)-(3.8).

We remark that when $\bar{F}=\bar{E}^{T}$, the above-discussed modified block unsymmetric Gauss-Seidel-type preconditioners naturally reduce to the modified block symmetric 
Gauss-Seidel-type (MBSGS-type) preconditioners to the matrix $A \in \mathbb{R}^{n \times n}$ in (1.2), correspondingly.

3.4. The case $S \approx-\bar{S}$. In the case that $\bar{S}$ is negative definite, we may let $S$ be an approximation to $-\bar{S}$ in order to obtain a preconditioner of positive definiteness in nature. Hence, some specified preconditioned Krylov subspace iteration method can exploit its efficiency sufficiently.

When $S \approx-\bar{S}$, for the MBJ-, the MBGS-, and the MBUGS-type preconditioners discussed above, we can demonstrate that the eigenvalues of the preconditioned matrices are, correspondingly, located within two circles having center $(-1,0)$ and $(1,0)$ in the complex plane. These results are precisely summarized in the following theorem. Since their proofs are essentially the same as those of Theorems 3.1, 3.3. 3.5 and 3.7 with only the identity matrix $I$ being replaced by the matrix

$$
J:=\left[\begin{array}{cc}
I & O \\
O & -I
\end{array}\right], \quad I \in \mathbb{R}^{p \times p} \quad \text { and } \quad-I \in \mathbb{R}^{q \times q},
$$

we only state the theorem but omit its proof.

Theorem 3.9. Let $M=P W Q \in \mathbb{R}^{n \times n}$ in (2.10) be the preconditioner to the block two-by-two matrix $A=P H Q \in \mathbb{R}^{n \times n}$ in (1.2), with $P$ and $Q$ being given by (2.6) and $H$ being given by (2.7). Let $K_{L}=W^{-1} H$ and $K_{R}=H W^{-1}$.

(i) If $W=W^{(J)}$ is defined by (3.3), then

$$
\left\|J-K_{L}\right\|_{2} \leq \rho_{L}^{(J)} \widetilde{\epsilon}_{L}, \quad\left\|J-K_{R}\right\|_{2} \leq \rho_{R}^{(J)} \widetilde{\epsilon}_{R},
$$

where $\rho_{L}^{(J)}$ and $\rho_{R}^{(J)}$ are the same as in Theorem 3.1.

(ii) If $W=W^{(G S)}$ is defined by (3.4), then

$$
\left\|J-K_{L}\right\|_{2} \leq \rho_{L}^{(G S)} \widetilde{\epsilon}_{L}, \quad\left\|J-K_{R}\right\|_{2} \leq \rho_{R}^{(G S)} \widetilde{\epsilon}_{R},
$$

where $\rho_{L}^{(G S)}$ and $\rho_{R}^{(G S)}$ are the same as in Theorem 3.3.

(iii) If $W=W^{(G S)}$ is defined by (3.5), then

$$
\left\|J-K_{L}\right\|_{2} \leq \rho_{L}^{(G S)} \widetilde{\epsilon}_{L}, \quad\left\|J-K_{R}\right\|_{2} \leq \rho_{R}^{(G S)} \widetilde{\epsilon}_{R},
$$

where $\rho_{L}^{(G S)}$ and $\rho_{R}^{(G S)}$ are the same as in Theorem 3.5.

(iv) If $W=W^{(U G S)}$ is defined by (3.6), then

$$
\left\|J-K_{L}\right\|_{2} \leq \rho_{L}^{(U G S)} \widetilde{\epsilon}_{L}, \quad\left\|J-K_{R}\right\|_{2} \leq \rho_{R}^{(U G S)} \widetilde{\epsilon}_{R},
$$

where $\rho_{L}^{(U G S)}$ and $\rho_{R}^{(U G S)}$ are the same as in Theorem 3.7.

It follows from Lemma 3.2 as well as (2.11) and (2.13) that the eigenvalues of the preconditioned matrix $M^{-1} A$ are located within the union of two circles having centers $(-1,0)$ and $(1,0)$ and radius $\rho_{L}^{(\xi)} \widetilde{\epsilon}_{L}$, and those of the preconditioned matrix $A M^{-1}$ are located within the union of two circles having centers $(-1,0)$ and $(1,0)$ and radius $\rho_{R}^{(\xi)} \widetilde{\epsilon}_{R}$, respectively. Therefore, they are all within $\widetilde{\mathcal{N}}^{(\xi)}$. Here, $\xi=J$, $G S$ and $U G S$.

We observe from the demonstrations of Theorems 3.1 3.9 that when $J_{B}=I$ or $S=\bar{S}$, the results in these theorems can be considerably improved and made more accurate. 
3.5. Connections to Krylov subspace methods. The preconditioning matrix $M$ defined in (2.10) can be used to accelerate the Krylov subspace methods such as GMRES or its restarted variant GMRES $(m)$ [1, 40, for solving the large sparse system of linear equations (1.1)-(1.2). This preconditioning matrix can be used as a left (see (2.11)-(2.12) ), a right (see (2.13)-(2.14)), or a split (see (2.15)-(2.17)) preconditioner to the system of linear equations (1.1). The obtained equivalent linear systems can be solved by GMRES or $\operatorname{GMRES}(m)$.

Assume that the coefficient matrices $\mathbf{A}$ of the above preconditioned linear systems are diagonalizable, i.e., there exist a nonsingular matrix $\mathbf{X} \in \mathbb{C}^{n \times n}$ and a diagonal matrix $\mathbf{D} \in \mathbb{C}^{n \times n}$ such that $\mathbf{A}=\mathbf{X D X}^{-1}$. Then it is well known from 41, Theorem 4] that the residual norm $\left\|\mathbf{r}^{(k)}\right\|_{2}$ at the $k$-th step of the preconditioned GMRES is bounded by $\left\|\mathbf{r}^{(k)}\right\|_{2} \leq \kappa(\mathbf{X}) \varepsilon^{(k)}\left\|\mathbf{r}^{(0)}\right\|_{2}$, where $\kappa(\mathbf{X})$ is the Euclidean condition number of $\mathbf{X}$ and $\varepsilon^{(k)}:=\min _{\mathcal{P} \in \mathcal{P}_{k}} \max _{\lambda_{i} \in \sigma(\mathbf{A})}\left|\mathcal{P}\left(\lambda_{i}\right)\right|$. Here, $\mathcal{P}_{k}$ denotes the set of all polynomials $\mathcal{P}(\lambda)$ of degree not greater than $k$ such that $\mathcal{P}(0)=1$, and $\sigma(\mathbf{A})$ denotes the spectrum of the matrix $\mathbf{A}$.

Consider $\bar{S}$ defined by (3.2); see also (2.1) and (2.3). When the matrix $S$ is an approximation to the matrix $\bar{S}$, from Theorems 3.1, 3.3, 3.5 and 3.7 we know that all eigenvalues of the matrix $\mathbf{A}$ are contained in either of the circles $\mathcal{N}^{(\xi)}, \xi=J$, $G S$ and $U G S$. Therefore, when $\rho^{(\xi)}<1$, a special case of Theorem 5 in 41 implies that $\varepsilon^{(k)} \leq\left(\rho^{(\xi)}\right)^{k}, \xi=J, G S$ and $U G S$.

Alternatively, the preconditioning matrix $M$ can also be used as a left, a right, or a split preconditioner to the system of linear equations (1.1) to obtain a preconditioned linear system of coefficient matrix $\widetilde{\mathbf{A}}=K_{L}, K_{R}$, or $K$, respectively. Because Theorems 3.2, 3.4, 3.6 and 3.8 guarantee the positive definiteness of the preconditioned matrix $\widetilde{\mathbf{A}}$, it is known from [20] and [41, p. 866] that the following error bound for the correspondingly preconditioned GMRES holds:

$$
\left\|\mathbf{r}^{(k)}\right\|_{2} \leq\left(1-\frac{\left(\lambda_{\min }(\widetilde{\mathbf{H}})\right)^{2}}{\lambda_{\max }\left(\widetilde{\mathbf{A}}^{T} \widetilde{\mathbf{A}}\right)}\right)^{\frac{k}{2}}\left\|\mathbf{r}^{(0)}\right\|_{2},
$$

where $\widetilde{\mathbf{H}}=\frac{1}{2}\left(\widetilde{\mathbf{A}}+\widetilde{\mathbf{A}}^{T}\right)$ denotes the symmetric part of the matrix $\widetilde{\mathbf{A}}$, and $\lambda_{\min }(\cdot)$ and $\lambda_{\max }(\cdot)$ denote, respectively, the smallest and the largest eigenvalues of the corresponding matrix. This gives a guarantee for the convergence of the restarted preconditioned GMRES iteration, say PGMRES $(m)$, for all $m$, when the coefficient matrix $\widetilde{\mathbf{A}}$ is positive definite.

When the matrix $S$ is an approximation to the matrix $-\bar{S}$, because the preconditioned matrix $\mathbf{A}$ or $\widetilde{\mathbf{A}}$ may be usually not positive definite, instead of GMRES and GMRES $(m)$ we may use other Krylov subspace methods such as BiCGSTAB, QMR and TFQMR to solve the preconditioned linear systems. In particular, when the original coefficient matrix $A$ is symmetric indefinite, MINRES is a possible candidate if a symmetric positive definite or indefinite preconditioner $M$ is obtainable. See [2, 27, 40].

\section{Applications to three typical matrices}

In this section, we will investigate the concretizations of the structured preconditioners established in Sections 2 and 3 to three special classes of matrices arising from real-world applications. 
4.1. The symmetric positive definite matrix. When the matrix blocks $B \in$ $\mathbb{R}^{p \times p}$ and $C \in \mathbb{R}^{q \times q}$ are symmetric positive definite, $F=E^{T}$ and the Schur complement $S_{A}=C-E^{T} B^{-1} E$ is symmetric positive definite, the matrix $A \in \mathbb{R}^{n \times n}$ reduces to the block two-by-two symmetric positive definite matrix

$$
A=\left[\begin{array}{cc}
B & E \\
E^{T} & C
\end{array}\right]
$$

These kinds of matrices may arise in the red/black ordering of a symmetric positive definite linear system, or in discretization incorporated with a domain decomposition technique of a boundary value problem of a self-adjoint elliptic partial differential equation, etc. See [2, 3, 6, 7, 27, 40].

Let $L_{B} \in \mathbb{R}^{p \times p}$ and $L_{C} \in \mathbb{R}^{q \times q}$ be nonsingular matrices such that either (2.1) or (2.2) holds with $R_{B}=L_{B}^{T}$ and $R_{C}=L_{C}^{T}$. Then from (2.10) and (2.6) we know that $M=P W Q$ is the structured preconditioner to the matrix $A$, where

$$
P=\left[\begin{array}{cc}
L_{B} & O \\
E^{T} L_{B}^{-T} & L_{C}
\end{array}\right], \quad Q=\left[\begin{array}{cc}
L_{B}^{T} & L_{B}^{-1} E \\
O & L_{C}^{T}
\end{array}\right]=P^{T},
$$

and $W \in \mathbb{R}^{n \times n}$ is an approximation to the matrix

$$
\bar{W}=\left[\begin{array}{cc}
J_{B} & \left(I-J_{B}\right) \bar{E} \\
\bar{E}^{T}\left(I-J_{B}\right) & \bar{S}
\end{array}\right] \approx H
$$

defined by (2.7), with $\bar{E}=L_{B}^{-1} E L_{C}^{-T}$ and $\bar{S}=J_{C}-\bar{E}^{T} \bar{E}$.

Note that $\bar{S}$ and $\bar{W}$ are symmetric positive definite. Let $S \in \mathbb{R}^{q \times q}$ be an approximation to the matrix $I-\bar{E}^{T} \bar{E} \approx \bar{S}$. To guarantee the symmetric positive definiteness of the preconditioning matrix $M$, we can choose $W$ to be the modified block Jacobi splitting matrix in (3.3) or the modified block symmetric Gauss-Seidel splitting matrix in (3.6), obtaining the modified block Jacobi-type preconditioner or the modified block symmetric Gauss-Seidel-type preconditioner to the matrix $A$, respectively.

4.2. The saddle point matrix. When the matrix block $B \in \mathbb{R}^{p \times p}$ is symmetric positive definite, $C=O$ and $F= \pm E^{T}$ is of full row rank, the matrix $A \in \mathbb{R}^{n \times n}$ reduces to the saddle point matrices

$$
A^{ \pm}=\left[\begin{array}{cc}
B & E \\
\pm E^{T} & O
\end{array}\right] .
$$

These kinds of matrices may arise in constrained optimization as well as leastsquares, saddle-point and Stokes problems, without a regularizing/stabilizing term, etc. See 14, 16, 24, 25, 28, 37, 44.

Let $L_{B} \in \mathbb{R}^{p \times p}$ be a nonsingular matrix such that either (2.1) or (2.2) holds with $R_{B}=L_{B}^{T}$ and $L_{C}=R_{C}=I$. Then from (2.10) and (2.6) we know that $M^{ \pm}=P^{ \pm} W^{ \pm} Q^{ \pm}$are the preconditioners to the matrices $A^{ \pm}$, respectively, where

$$
P^{ \pm}=\left[\begin{array}{cc}
L_{B} & O \\
\pm E^{T} L_{B}^{-T} & I
\end{array}\right], \quad Q^{ \pm}=\left[\begin{array}{cc}
L_{B}^{T} & L_{B}^{-1} E \\
O & I
\end{array}\right],
$$


and $W^{ \pm} \in \mathbb{R}^{n \times n}$ are approximations to the matrices

$$
\bar{W}^{ \pm}=\left[\begin{array}{cc}
J_{B} & \left(I-J_{B}\right) \bar{E} \\
\pm \bar{E}^{T}\left(I-J_{B}\right) & \bar{S}^{ \pm}
\end{array}\right] \approx H^{ \pm}
$$

defined by (2.7), with $\bar{E}=L_{B}^{-1} E$ and $\bar{S}^{ \pm}=\mp \bar{E}^{T} \bar{E}$.

Let $S^{ \pm} \in \mathbb{R}^{q \times q}$ be approximations to the matrices $\bar{S}^{ \pm}$. By choosing the matrices $W^{ \pm}$to be the modified block Jacobi splitting matrices in (3.3), the modified block Gauss-Seidel splitting matrices in (3.4) or (3.5), or the modified block unsymmetric Gauss-Seidel splitting matrices in (3.6), we can obtain the modified block Jacobitype preconditioners, the modified block Gauss-Seidel-type preconditioners, or the modified block unsymmetric Gauss-Seidel-type preconditioners to the matrices $A^{ \pm}$, respectively.

4.3. The Hamiltonian matrix. When the matrix block $B \in \mathbb{R}^{p \times p}$ is symmetric positive definite and $C \in \mathbb{R}^{q \times q}$ is symmetric positive/negative definite (denoted by $C_{+} / C_{-}$, respectively), and $F=\mp E^{T}$, the matrix $A \in \mathbb{R}^{n \times n}$ reduces to the Hamiltonian matrices

$$
A^{ \pm}=\left[\begin{array}{cc}
B & E \\
\mp E^{T} & C_{ \pm}
\end{array}\right] \text {. }
$$

These kinds of matrices may arise in stationary semiconductor devices [36, 43, 42, in constrained optimization as well as least-squares, saddle-point and Stokes problems, with a regularizing/stabilizing term 28 .

Let $L_{B} \in \mathbb{R}^{p \times p}$ and $L_{C_{ \pm}} \in \mathbb{R}^{q \times q}$ be nonsingular matrices such that either (2.1) or (2.2) holds with $R_{B}=L_{B}^{T}$ and $R_{C_{ \pm}}=L_{C_{ \pm}}^{T}$. Then from (2.10) and (2.6) we know that $M^{ \pm}=P^{ \pm} W^{ \pm} Q^{ \pm}$are the preconditioners to the matrices $A^{ \pm}$, where

$$
P^{ \pm}=\left[\begin{array}{cc}
L_{B} & O \\
\mp E^{T} L_{B}^{-T} & L_{C_{ \pm}}
\end{array}\right], \quad Q^{ \pm}=\left[\begin{array}{cc}
L_{B}^{T} & L_{B}^{-1} E \\
O & L_{C_{ \pm}}^{T}
\end{array}\right],
$$

and $W^{ \pm} \in \mathbb{R}^{n \times n}$ are approximations to the matrices

$$
\bar{W}^{ \pm}=\left[\begin{array}{cc}
J_{B} & \left(I-J_{B}\right) \bar{E}^{ \pm} \\
\mp\left(\bar{E}^{ \pm}\right)^{T}\left(I-J_{B}\right) & \bar{S}^{ \pm}
\end{array}\right] \approx H^{ \pm}
$$

defined by (2.7), with $\bar{E}^{ \pm}=L_{B}^{-1} E L_{C_{ \pm}}^{-T}$ and $\bar{S}^{ \pm}=J_{C} \pm\left(\bar{E}^{ \pm}\right)^{T} \bar{E}^{ \pm}$.

Let $S^{ \pm} \in \mathbb{R}^{q \times q}$ be approximations to the matrices $I \pm\left(\bar{E}^{ \pm}\right)^{T} \bar{E}^{ \pm} \approx \bar{S}^{ \pm}$. By choosing the matrices $W^{ \pm}$to be the modified block Jacobi splitting matrices in (3.3), the modified block Gauss-Seidel splitting matrices in (3.4) or (3.5), or the modified block unsymmetric Gauss-Seidel splitting matrices in (3.6), we can obtain the modified block Jacobi-type preconditioners, the modified block Gauss-Seidel-type preconditioners, or the modified block unsymmetric Gauss-Seidel-type preconditioners to the matrices $A^{ \pm}$, respectively.

4.4. An illustrative example. Let us consider the electromagnetic scattering problem from a large rectangular cavity on the $(x, y)$-plane in which the medium is $y$-directional inhomogeneous. In the transverse magnetic polarization case, when the model Helmholtz equation with positive wave number is discretized by the fivepoint finite difference scheme with uniform stepsize $h$, we obtain a block two-by-two system of linear equations (1.1)-(1.2), in which

$$
B=V \otimes I+I \otimes V-I \otimes \Omega \in \mathbb{R}^{p \times p}, \quad C=I-h G \in \mathbb{R}^{q \times q}, \quad E=I \otimes e_{q} \in \mathbb{R}^{p \times q}
$$


and $F=-E^{T}$, where $h=\frac{1}{q+1}, p=q^{2}, \theta \geq 0$ is a real constant, $e_{q}$ is the $q$-th unit vector in $\mathbb{R}^{q}, I$ is the $q$-by- $q$ identity matrix, $V=\operatorname{tridiag}\left(-1+\frac{1}{2} \theta h, 2,-1-\frac{1}{2} \theta h\right) \in$ $\mathbb{R}^{q \times q}$ is a tridiagonal matrix, $\Omega=h^{2} \cdot \operatorname{diag}\left(\omega_{1}^{2}, \omega_{2}^{2}, \ldots, \omega_{q}^{2}\right) \in \mathbb{R}^{q \times q}$ is a nonnegative diagonal matrix, $G=\left(g_{i j}\right) \in \mathbb{R}^{q \times q}$, and $\otimes$ denotes the Kronecker product. See [33, 1].

Concretely, in our computations we take $\theta=1, \omega_{i}=16 \pi(i=1,2, \ldots, q)$, and $g_{i j}=\frac{1}{(i+j)^{2}}(i, j=1,2, \ldots, q)$.

Let $B \approx L_{B} R_{B}$ be an incomplete triangular factorization of the matrix block $B$, and $L_{C}=R_{C}=I$. Then we have

$$
\bar{E}=L_{B}^{-1} E, \quad \bar{F}=-E^{T} R_{B}^{-1}, \quad J_{C}=C \quad \text { and } \quad \bar{S}=C-\overline{F E} .
$$

Now, by choosing $S=$ band $_{\ell_{b}}(C)-\overline{F E}$ with band , $_{b}(C)$ being the band matrix of half-band width $\ell_{b}$ truncated from the matrix $C$, after straightforward computations we can obtain the results listed in Tables 1-4 for the discretization stepsizes $h=\frac{1}{16}$, $\frac{1}{24}, \frac{1}{32}$ and $\frac{1}{64}$, or equivalently, for the problem sizes $(p, q)=(225,15),(529,23)$, $(961,31)$ and $(3969,63)$, respectively.

In Table 1 we list the half-band width $\ell_{b}$, the quantities

$$
\Theta=\|\bar{E}\|_{2}, \quad \Gamma=\|\bar{F}\|_{2}, \quad \Theta_{s}=\left\|\bar{E} S^{-1}\right\|_{2} \quad \text { and } \quad \Gamma_{s}=\left\|S^{-1} \bar{F}\right\|_{2}
$$

with respect to the matrix norms, and

$$
\epsilon_{L}=\max \left\{\left\|I-J_{B}\right\|_{2},\left\|I-S^{-1} \bar{S}\right\|_{2}\right\} \quad \text { and } \quad \epsilon_{R}=\max \left\{\left\|I-J_{B}\right\|_{2},\left\|I-\bar{S} S^{-1}\right\|_{2}\right\}
$$

with respect to the matrix approximation accuracies. For $\xi=J, G S$ and $U G S$, in Tables 2-4 we list the radii $\rho_{L}^{(\xi)} \epsilon_{L}$ and $\rho_{R}^{(\xi)} \epsilon_{R}$ of the circles centered at $(1,0)$ where all eigenvalues of the matrices $K_{L}$ and $K_{R}$ are located within $\rho^{(\xi)}=$ $\min \left\{\rho_{L}^{(J)} \epsilon_{L}, \rho_{R}^{(J)} \epsilon_{R}\right\}$ and the radii $\rho_{*}^{(\xi)}$ of the smallest circles that include all eigenvalues of the corresponding preconditioned matrices (see Theorems 3.1] 3.3] and 3.7), and the quantities $\delta_{L}^{(\xi)}$ and $\delta_{R}^{(\xi)}$ that guarantee the positive definiteness of the preconditioned matrices $K_{L}$ and $K_{R}$ whenever $\epsilon_{L}<\delta_{L}^{(\xi)}$ and $\epsilon_{R}<\delta_{R}^{(\xi)}$ (see Theorems 3.2, 3.4 and 3.8), respectively.

TABLE 1. Quantities with respect to the preconditioned matrices

\begin{tabular}{|c|c|c|c|c|}
\hline$h$ & $\frac{1}{16}$ & $\frac{1}{24}$ & $\frac{1}{32}$ & $\frac{1}{64}$ \\
\hline$\ell_{b}$ & 2 & 4 & 6 & 30 \\
\hline$\Theta$ & 11.9704 & 15.8432 & 25.3679 & 28.8844 \\
\hline$\Gamma$ & 6.05339 & 7.38068 & 8.95453 & 14.7829 \\
\hline$\Theta_{s}$ & 5.77108 & 6.57523 & 11.0775 & 39.2410 \\
\hline$\Gamma_{s}$ & 2.82323 & 3.28938 & 3.93560 & 19.5354 \\
\hline$\epsilon_{L}$ & $2.95 e-03$ & $1.52 e-03$ & $6.39 e-04$ & $5.75 e-05$ \\
\hline$\epsilon_{R}$ & $4.13 e-03$ & $2.15 e-03$ & $7.89 e-04$ & $4.63 e-05$ \\
\hline
\end{tabular}


TABLE 2. Bounds with respect to the MBJ-type preconditioner $M=P W^{(J)} Q$ with $W^{(J)}$ being defined by (3.3)

\begin{tabular}{|c|c|c|c|c|}
\hline$h$ & $\frac{1}{16}$ & $\frac{1}{24}$ & $\frac{1}{32}$ & $\frac{1}{64}$ \\
\hline$\rho_{L}^{(J)} \epsilon_{L}$ & 0.111566 & $8.62 \mathrm{e}-02$ & $6.78 \mathrm{e}-02$ & $3.26 \mathrm{e}-02$ \\
\hline$\rho_{R}^{(J)} \epsilon_{R}$ & 0.152507 & 0.108497 & $7.98 \mathrm{e}-02$ & $2.70 \mathrm{e}-02$ \\
\hline$\rho^{(J)}$ & 0.111566 & $8.62 \mathrm{e}-02$ & $6.78 \mathrm{e}-02$ & $2.70 \mathrm{e}-02$ \\
\hline$\delta_{L}^{(J)}$ & $2.75 e-02$ & $1.82 e-02$ & $9.71 e-03$ & $1.77 e-02$ \\
\hline$\delta_{R}^{(J)}$ & $2.71 e-02$ & $1.98 e-02$ & $9.88 e-03$ & $1.72 e-03$ \\
\hline$\rho_{*}^{(J)}$ & $2.31 e-03$ & $1.24 e-03$ & $4.63 e-04$ & $3.93 e-05$ \\
\hline
\end{tabular}

TABLE 3. Bounds with respect to the MBGS-type preconditioner $M=P W^{(G S)} Q$ with $W^{(G S)}$ being defined by (3.4)

\begin{tabular}{|c|c|c|c|c|}
\hline$h$ & $\frac{1}{16}$ & $\frac{1}{24}$ & $\frac{1}{32}$ & $\frac{1}{64}$ \\
\hline$\rho_{L}^{(G S)} \epsilon_{L}$ & $3.55 e-02$ & $2.41 e-02$ & $1.62 e-02$ & $1.66 e-03$ \\
\hline$\rho_{R}^{(G S)} \epsilon_{R}$ & $2.45 e-02$ & $1.44 e-02$ & $8.81 e-03$ & $1.82 e-03$ \\
\hline$\rho^{(G S)}$ & $2.45 e-02$ & $1.44 e-02$ & $8.81 e-03$ & $1.66 e-03$ \\
\hline$\delta_{L}^{(G S)}$ & $2.69 e-02$ & $1.79 e-02$ & $9.63 e-03$ & $1.76 e-03$ \\
\hline$\delta_{R}^{(G S)}$ & $7.60 e-03$ & $4.69 e-03$ & $1.97 e-03$ & $2.17 e-04$ \\
\hline$\rho_{*}^{(G S)}$ & $2.31 e-03$ & $1.24 e-03$ & $4.63 e-04$ & $3.93 e-05$ \\
\hline
\end{tabular}

TABLE 4. Bounds with respect to the MBUGS-type preconditioner $M=P W^{(U G S)} Q$ with $W^{(U G S)}$ being defined by (3.6)

\begin{tabular}{|c|c|c|c|c|}
\hline$h$ & $\frac{1}{16}$ & $\frac{1}{24}$ & $\frac{1}{32}$ & $\frac{1}{64}$ \\
\hline$\rho_{L}^{(U G S)} \epsilon_{L}$ & 0.102518 & $8.06 e-02$ & 0.166135 & $3.25 e-02$ \\
\hline$\rho_{R}^{(U G S)} \epsilon_{R}$ & 0.148479 & 0.106365 & 0.216704 & $2.69 e-02$ \\
\hline$\rho^{(U G S)}$ & 0.102518 & $8.06 e-02$ & 0.166135 & $2.69 e-02$ \\
\hline$\delta_{L}^{(U G S)}$ & $3.62 e-03$ & $1.87 e-03$ & $6.54 e-04$ & $7.16 e-05$ \\
\hline$\delta_{R}^{(U G S)}$ & $4.32 e-03$ & $2.69 e-03$ & $9.47 e-04$ & $6.25 e-05$ \\
\hline$\rho_{*}^{(U G S)}$ & $2.31 e-03$ & $1.24 e-03$ & $4.63 e-04$ & $3.93 e-05$ \\
\hline
\end{tabular}

The results in Tables 2-4 clearly show that

(i) for $\xi=J, G S$ and $U G S, \rho_{L}^{(\xi)} \epsilon_{L}<1$ and $\rho_{R}^{(\xi)} \epsilon_{R}<1$. It follows that $\rho^{(\xi)}<1$. As $\rho_{L}^{(\xi)} \epsilon_{L}$ and $\rho_{R}^{(\xi)} \epsilon_{R}$ are quite small, the eigenvalues of the preconditioned matrices, with respect to the MBJ-, the MBGS- and the MBUGS-type preconditioners, are tightly clustered around the point $(1,0)$; see Theorems 3.1, 3.3 and 3.7. Hence, a Krylov subspace method such as GMRES, when applied to the preconditoned systems of linear equations, will achieve fast convergence; see Section 3.5.

(ii) for $\xi=J$, GS and $U G S, \epsilon_{L}<\delta_{L}^{(\xi)}<1$ and $\epsilon_{R}<\delta_{R}^{(\xi)}<1$. It follows that the preconditioned matrices, with respect to the MBJ-, the MBGS- and the 
MBUGS-type preconditioners, are positive definite, and the convergence of the restarted GMRES methods preconditioned by these preconditioners are guaranteed; see Theorems 3.23 .4 and 3.8 as well as Section 3.5.

(iii) for $\xi=J, G S$ and $U G S, \rho_{*}^{(\xi)} \leq \rho^{(\xi)}$. This shows that the eigenvalues of the preconditioned matrices, with respect to the MBJ-, the MBGS- and the MBUGS-type preconditioners, are really located within the theoretically estimated circles centered at $(1,0)$ with radii $\rho^{(\xi)}$ given in Theorems 3.1 . 3.3 and 3.7, respectively.

In summary, this example shows that the conditions of our theorems are reasonable and the conclusions of them are correct.

\section{CONClusion AND Remarks}

We have established a general framework of practical and efficient structured preconditioners to the large sparse block two-by-two nonsingular matrices. For several special cases associated with the modified block relaxation iteration methods, we have studied the eigenvalue distributions and the positive definiteness of the preconditioned matrices. Theoretical analyses have shown that this preconditioning technique can afford effective and high-quality preconditioners to the Krylov subspace iteration methods for solving large sparse systems of linear equations with block two-by-two coefficient matrices.

We remark that our preconditioning technique and the corresponding theory can be straightforwardly developed to the following cases.

(a) The approximation matrix $W \in \mathbb{R}^{n \times n}$ in (2.10) that is generated by a multi-step variant of the modified block Jacobi, the modified block GaussSeidel or the modified block unsymmetric Gauss-Seidel splitting matrix of the matrix $\bar{W} \in \mathbb{R}^{n \times n}$ in (2.7) [6, 7].

(b) Alternatively, the approximation matrix $W \in \mathbb{R}^{n \times n}$ in (2.10) that is generated by a single- or multiple-step variant of the modified block successive overrelaxation (SOR), the modified block unsymmetric SOR, the modified block accelerated overrelaxation (AOR) or the modified block unsymmetric AOR splitting matrix of the matrix $\bar{W} \in \mathbb{R}^{n \times n}$ in (2.7) 32, 6, 7].

(c) More generally, the approximation matrix $W \in \mathbb{R}^{n \times n}$ in (2.10) that is generated by any suitable direct or iterative method induced by the matrix $\bar{W} \in \mathbb{R}^{n \times n}$ in (2.7).

(d) The matrix $A \in \mathbb{R}^{n \times n}$ that is of a general $\ell$-by- $\ell$ block structure. More concretely, $A=\left(A_{i, j}\right) \in \mathbb{R}^{n \times n}$, where $A_{i, j} \in \mathbb{R}^{n_{i} \times n_{j}}, i, j=1,2, \ldots, \ell$, and $n_{i}(i=1,2, \ldots, \ell)$ are positive integers satisfying $n_{1}+n_{2}+\ldots+n_{\ell}=n$.

For the structured preconditioners based on the relaxation iteration methods involving parameters, we can further optimize them through choices of the optimal parameters. In addition, we should point out that, although all results in this paper are demonstrated in the $\|\cdot\|_{2}$-norm, they trivially hold for other consistent matrix norms such as the $\|\cdot\|_{1}$-norm and the $\|\cdot\|_{\infty}$-norm.

\section{ACKNOWLEDGMENTS}

The author is very much indebted to the referees for their constructive and valuable comments and suggestions which greatly improved the original version of this paper. 


\section{REFERENCES}

[1] H. Ammari, G. Bao and A.W. Wood, An integral equation method for the electromagnetic scattering from cavities, Math. Methods Appl. Sci., 23(2000), 1057-1072. MR 1773922 (2001g:78013)

[2] O. Axelsson, Iterative Solution Methods, Cambridge University Press, Cambridge, 1994. MR.1276069 (95f:65005)

[3] Z.-Z. Bai, Parallel Iterative Methods for Large-Scale Systems of Algebraic Equations, Ph.D. Thesis of Shanghai University of Science and Technology, Shanghai, June 1993. (In Chinese)

[4] Z.-Z. Bai, A class of hybrid algebraic multilevel preconditioning methods, Appl. Numer. Math., 19(1996), 389-399. MR1377785|(96j:65116)

[5] Z.-Z. Bai, Parallel hybrid algebraic multilevel iterative methods, Linear Algebra Appl., 267(1997), 281-315. MR1479124 (99c:65081)

[6] Z.-Z. Bai, A class of modified block SSOR preconditioners for symmetric positive definite systems of linear equations, Adv. Comput. Math., 10(1999), 169-186. MR.1680610(2000c:65020)

[7] Z.-Z. Bai, Modified block SSOR preconditioners for symmetric positive definite linear systems, Ann. Operations Research, 103(2001), 263-282. MR.1868455 (2002k:65046)

[8] Z.-Z. Bai, I.S. Duff and A.J. Wathen, A class of incomplete orthogonal factorization methods. I: methods and theories, BIT, 41(2001), 53-70. MR1829661(2002b:65040)

[9] Z.-Z. Bai and G.-Q. Li, Restrictively preconditioned conjugate gradient methods for systems of linear equations, IMA J. Numer. Anal., 23(2003), 561-580. MR2011340(2004i:65025)

[10] Z.-Z. Bai and M.K. Ng, On inexact preconditioners for nonsymmetric matrices, SIAM J. Sci. Comput., 26(2005), 1710-1724.

[11] Z.-Z. Bai and Z.-Q. Wang, Restrictive preconditioners for conjugate gradient methods for symmetric positive definite linear systems, J. Comput. Appl. Math., 187(2006), 202-226.

[12] Z.-Z. Bai and D.-R. Wang, A class of new hybrid algebraic multilevel preconditioning methods, Linear Algebra Appl., 260(1997), 223-255. MR.1448358 (98i:65035)

[13] J.T. Betts, Practical Methods for Optimal Control Using Nonlinear Programming, SIAM, Philadelphia, PA, 2001. MR.1826768 (2002e:49001)

[14] A. Björck, Numerical Methods for Least Squares Problems, SIAM, Philadelphia, PA, 1996. MR.1386889 (97g:65004)

[15] J.H. Bramble, J.E. Pasciak and A.T. Vassilev, Analysis of the inexact Uzawa algorithm for saddle point problems, SIAM J. Numer. Anal., 34(1997), 1072-1092. MR1451114|(98c:65182)

[16] F. Brezzi and M. Fortin, Mixed and Hybrid Finite Element Methods, Springer-Verlag, New York, 1991. MR1115205(92d:65187)

[17] I.S. Duff, N.I.M. Gould, J.K. Reid, J.A. Scott and K. Turner, The factorization of sparse symmetric indefinite matrices, IMA J. Numer. Anal., 11(1991), 181-204. MR.1105227(92a:65143)

[18] I.S. Duff and J.K. Reid, Exploiting zeros on the diagonal in the direct solution of indefinite sparse symmetric linear systems, ACM Trans. Math. Software, 22(1996), 227-257. MR.1408491 (97c:65085)

[19] N. Dyn and W.E. Ferguson, Jr., The numerical solution of equality constrained quadratic programming problems, Math. Comput., 41(1983), 165-170. MR0701631 (85b:90051)

[20] S.C. Eisenstat, H.C. Elman and M.H. Schultz, Variational iterative methods for nonsymmetric systems of linear equations, SIAM J. Numer. Anal., 20(1983), 345-357. MR0694523 (84h:65030)

[21] H.C. Elman, Preconditioners for saddle point problems arising in computational fluid dynamics, Appl. Numer. Math., 43(2002), 75-89. MR1936103

[22] H.C. Elman and G.H. Golub, Inexact and preconditioned Uzawa algorithms for saddle point problems, SIAM J. Numer. Anal., 31(1994), 1645-1661. MR.1302679 (95f:65065)

[23] H.C. Elman, D.J. Silvester and A.J. Wathen, Performance and analysis of saddle point preconditioners for the discrete steady-state Navier-Stokes equations, Numer. Math., 90(2002), 665-688. MR 1888834(2002m:76071)

[24] B. Fischer, R. Ramage, D.J. Silvester and A.J. Wathen, Minimum residual methods for augmented systems, BIT, 38(1998), 527-543. MR1652781 (99i:65031)

[25] P.E. Gill, W. Murray and M.H. Wright, Practical Optimization, Academic Press, New York, NY, 1981. MR0634376 (83d:65195)

[26] R. Glowinski, Numerical Methods for Nonlinear Variational Problems, Springer-Verlag, New York, 1984. MR0737005(86c:65004) 
[27] G.H. Golub and C.F. Van Loan, Matrix Computations, 3rd Edition, The Johns Hopkins University Press, Baltimore and London, 1996. MR.1417720 (97g:65006)

[28] G.H. Golub and A.J. Wathen, An iteration for indefinite systems and its application to the Navier-Stokes equations, SIAM J. Sci. Comput., 19(1998), 530-539. MR1618828 (99d:65107)

[29] G.H. Golub, X. Wu and J.-Y. Yuan, SOR-like methods for augmented systems, BIT, 41(2001), 71-85. MR:1829662(2002a:65055)

[30] N.I.M. Gould, M.E. Hribar and J. Nocedal, On the solution of equality constrained quadratic programming problems arising in optimization, SIAM J. Sci. Comput., 23(2001), 1375-1394. MR1885606 (2002k:90072)

[31] E. Haber, U.M. Ascher and D. Oldenburg, On optimization techniques for solving nonlinear inverse problems, Inverse Problems, 16(2000), 1263-1280. MR.1798355(2001j:78025)

[32] A. Hadjidimos, Accelerated overrelaxation method, Math. Comput., 32(1978), 149-157. MR0483340 (58:3353)

[33] J. Jin, The Finite Element Method in Electromagnetics, John Wiley $\&$ Sons, New York, NY, 1993. MR1903357 (2004b:78019)

[34] C. Keller, N.I.M. Gould and A.J. Wathen, Constrained preconditioning for indefinite linear systems, SIAM J. Matrix Anal. Appl., 21(2000), 1300-1317. MR.1780274 (2002b:65050)

[35] A. Klawonn, Block-triangular preconditioners for saddle point problems with a penalty term, SIAM J. Sci. Comput., 19(1998), 172-184. MR.1616885 (99f:65051)

[36] P.A. Markowich, The Stationary Semiconductor Device Equations, Springer-Verlag, New York, 1986. MR0821965 (87b:78042)

[37] M.F. Murphy, G.H. Golub and A.J. Wathen, A note on preconditioning for indefinite linear systems, SIAM J. Sci. Comput., 21(2000), 1969-1972. MR.1762024(2001a:65055)

[38] I. Perugia and V. Simoncini, Block-diagonal and indefinite symmetric preconditioners for mixed finite element formulations, Numer. Linear Algebra Appl., 7(2000), 585-616. MR:1802361 (2001j:65187)

[39] R.J. Plemmons, A parallel block iterative method applied to computations in structural analysis, SIAM J. Alg. Disc. Meth., 7(1986), 337-347. MR0844035 (88h:49058)

[40] Y. Saad, Iterative Methods for Sparse Linear Systems, 2nd Edition, SIAM, Philadelphia, PA, 2003. MR 1990645 (2004h:65002)

[41] Y. Saad and M.H. Schultz, GMRES: a generalized minimal residual algorithm for solving nonsymmetric linear systems, SIAM J. Sci. Stat. Comput., 7(1986), 856-869. MR0848568 (87g:65064)

[42] G.E. Sartoris, A 3D rectangular mixed finite element method to solve the stationary semiconductor equations, SIAM J. Sci. Stat. Comput., 19(1998), 387-403. MR1618875 (99b:65147)

[43] S. Selberherr, Analysis and Simulation of Semiconductor Devices, Springer-Verlag, New York, 1984.

[44] G. Strang, Introduction to Applied Mathematics, Wellesley-Cambridge Press, MA, 1986. MR0870634 (88a:00006)

[45] W. Zulenher, Analysis of iterative methods for saddle point problems: a unified approach, Math. Comput., 71(2001), 479-505. MR1885611 (2003f:65183)

State Key Laboratory of Scientific/Engineering Computing, Institute of Computational Mathematics and Scientific/Engineering Computing, Academy of Mathematics and System Sciences, Chinese Academy of Sciences, P.O. Box 2719, Beijing 100080, PeoPLE'S REPUBLIC OF CHINA

E-mail address: bzz@lsec.cc.ac.cn 